\title{
Algorithms for the partitioned solution of weakly coupled fluid models for cardiovascular flows
}

\author{
A. Cristiano I. Malossi ${ }^{1, *, \dagger}$, Pablo J. Blanco ${ }^{2,3}$, Simone Deparis ${ }^{1}$ and \\ Alfio Quarteroni ${ }^{1,4}$ \\ ${ }^{1}$ CMCS, Chair of Modelling and Scientific Computing, MATHICSE, Mathematics Institute of Computational Science \\ and Engineering, EPFL, École Polytechnique Fédérale de Lausanne, Station 8, CH-1015, Lausanne, Switzerland \\ ${ }^{2}$ LNCC, Laboratório Nacional de Computação Científica, Av. Getúlio Vargas 333, Quitandinha, 25651-075, \\ Petrópolis, Brazil \\ ${ }^{3}$ INCT-MACC, Instituto Nacional de Ciência e Tecnologia em Medicina Assistida por Computação Científica, \\ Petrópolis, Brazil \\ ${ }^{4}$ MOX, Modeling and Scientific Computing, Department of Mathematics, Politecnico di Milano, Via Bonardi 9 , \\ Milan, Italy
}

\begin{abstract}
SUMMARY
The main goal of this work is to devise robust iterative strategies to partition the solution of the Navier-Stokes equations in a three-dimensional (3D) domain, into non-overlapping 3D subdomains, which communicate through the exchange of averaged/integrated quantities across the interfaces. The novel aspect of the present approach is that at coupling boundaries, the conservation of flow rates and of the associated dual variables is implicitly imposed, entailing a weak physical coupling. For the solution of the non-linear interface problem two strategies are compared: relaxed fixed-point and Newton iterations. The algorithm is tested in several configurations for problems ranging from academic ones to some related to the computational haemodynamics field, which involve more than two components at each coupling interface. In some cases, it is shown that relaxed fixed-point methods are not convergent, whereas the Newton method leads in all tested cases to convergent schemes. One of the appealing aspects of the strategy proposed here is the flexibility in the setting of boundary conditions at the interfaces, where no hierarchy is established a priori (unlike Gauss-Seidel methods). The usefulness of this methodology is also discussed in the context of dimensionally heterogeneous coupling and preconditioning for domain decomposition methods. Copyright (C) 2011 John Wiley \& Sons, Ltd.
\end{abstract}

Received 4 March 2011; Revised 24 May 2011; Accepted 24 May 2011

KEY WORDS: domain decomposition; parallel algorithms; Navier-Stokes equations; geometrical multiscale; Newton method; haemodynamics

\section{INTRODUCTION}

In the last years, there have been increasing efforts towards performing the coupling of models that are heterogeneous regarding some of their features. In specific applications, this has been proven by the increasing use of dimensionally heterogeneous coupled models with the aim of including, in the global descriptions of the problem, features pertaining to different geometrical scales.

In the field of computational haemodynamics, dimensional heterogeneity of the constituent models, or components, is mandatory to correctly model the global and local circulations. On that point, the use of coupled three-dimensional (3D) or two-dimensional (2D) detailed models based

\footnotetext{
*Correspondence to: A. Cristiano I. Malossi, Chair of Modelling and Scientific Computing, École Polytechnique Fédérale de Lausanne, Av. Piccard, Station 8, CH-1015, Lausanne, Switzerland.

$\dagger$ E-mail: cristiano.malossi@epfl.ch 
on the Navier-Stokes equations with one-dimensional (1D) and/or zero-dimensional (0D) models to account for the surrounding part of the geometrically isolated vessel has become common practice [1-9]. Either imposing a defective boundary condition [10-12], setting a kinematicallyincompatible variational problem $[1,13]$ or imposing a lumped impedance boundary condition $[5,9]$ leads to imposing constraints over the flow rate across the inlets/outlets of the computational domain modelled by the Navier-Stokes equations.

Nonetheless, at discrete level, these continuity equations couple a large number of degrees of freedom, posing some practical difficulties at the discrete level. This motivates the development of iterative strategies in order to deal, in a segregated manner, with such coupled models. Let us recall that the continuity conditions enforced between these heterogeneous models are written in terms of averaged/integrated quantities that somehow belong to the lower-dimensional 0D/1D models, as showed in the examples cited in the previous paragraph.

In the computational haemodynamics community, the efforts made so far to decouple this problem into homogeneous subproblems have been addressed in very specific situations, without attempting to develop an abstract setting (see approaches in $[1,3,7,10]$ ). More recently, Leiva et al. [14] proposed dealing with this kind of coupling by rewriting it as an interface problem for which any matrix-free method for linear systems can be applied (in the case of linear problems). This interface problem is characterized by having a very small number of degrees of freedom because the interface unknowns are the 0D/1D quantities. In addition, another interesting aspect of that approach is that two unknowns per coupling interface are kept in the formulation, namely the primal and the dual variables of the problem. Here, primal and dual variables must be understood in the sense of the duality pairing representing the virtual power exerted between such pair of variables in a variational problem. This makes the decomposition into subproblems an easier task because it is possible to set up in an independent manner the boundary conditions for the models sharing a common coupling interface. In [15], this has also been successfully applied to the decomposition of 1D networks in order to simulate wave propagation phenomena in compliant vessels, addressing the simulation of the whole systemic circulation.

Exploiting the ideas developed in [14] and further extended in [15], the goal of the present work is to devise robust iterative strategies for coupling of dimensionally homogeneous flow models through averaged/integrated coupling quantities; these quantities are defined according to lower-dimensional fluid models and therefore provide a weak coupling in the case of 3D Navier-Stokes equations. The aim is to partition a 3D computational domain into several complementary non-overlapping subdomains, imposing, at each interface, the conservation of flow rate and the continuity of the associated dual variable, that is, the normal component of the traction vector. On the reduced problem involving the unknown interface variables, we apply the Newton method, which requires the evaluation of the Jacobian matrix, as well as a relaxed fixed-point method based on the Aitken acceleration. In [14, 15], a finite difference approximation is used to solve the Jacobian system; here, we propose to assemble the exact Jacobian matrix by exploiting the local tangent problems associated with the subdomains. Furthermore, the formulation and iterative solution of interface problems involving more than two subdomains merging at a coupling point is addressed. Hence, the developments of Leiva et al. [14] are not only applied for the problem studied here, but also generalized. This is the situation that arises, for instance, at bifurcations or trifurcations. In such cases, it is shown that neither the classical Aitken method nor its variants converge, whereas the Newton method leads, in all the tested cases, to convergent schemes. Another appealing aspect of this approach is the flexibility in the setting of the boundary conditions at the coupling interfaces, where no hierarchy should be established a priori (see [14]). Indeed, Gauss-Seidel methods define a sequential exchange of information at the interfaces, thus yielding an undesirable hierarchical interaction between the models.

All the features of the coupling strategy proposed here are shown through several examples, ranging from academic examples to situations in computational haemodynamics. The purpose of the examples is twofold. On the one hand, they are used to give a detailed account of how the approach is dealt with, in a practical way. On the other hand, they are employed to give numerical evidences of the robustness of the approach in contrast to the poor convergence properties of Aitken-based methodologies. 
It is also worth pointing out that these partitioned strategies are by no means restricted to the specific problem studied here, and this is why they are kept as general as possible, so they can be applied to other situations as follows: (i) the coupling of dimensionally heterogeneous models just by replacing some of the 3D models by 1D (or 0D) condensed models; or (ii) as preconditioners when solving the classical domain decomposition problem involving the Navier-Stokes equations.

This paper is organized as follows: in Section 2, we introduce the general approach for the weak coupling of fluid flow models, which is addressed through two different strategies. Then, in Section 3; we solve the coupled problem using two different approaches: the Aitken method and the Newton method. These techniques are applied to three numerical examples presented in Section 4, whereas in Section 5, we discuss other extensions and fields of application of the ideas developed here. Finally, in Section 6, we conclude briefly summarizing the main results.

\section{DESCRIPTION OF THE PROBLEM}

In this section, we propose a general approach for the weak coupling of fluid flow models. The main field of application of the methodology devised here is the modelling of complex cardiovascular networks, but it can also be employed to deal with other problems, such as the study of fluid/gas flows in pipe networks (see for instance $[16,17]$ ) and structural frames in solid mechanics (see $[18,19]$ and the references therein), among others. As aforesaid in the introduction, the driving motivation is to investigate the setup and computational implementation of a decomposition methodology when aimed at a geometrical multiscale framework, in such a way that it will be possible to assemble a network through the contribution of components of different geometrical dimension (i.e., 0D, 1D and 3D) and governed by partial differential equations (PDEs) of various nature. One of the main issues of this problem is how to couple together different models, through heterogeneous interfaces, without losing generality. Such framework has to be flexible enough to assemble a network of heterogeneous (or homogeneous) elements using a general setting. This objective is achieved by imposing the conservation of averaged/integrated quantities over the interfaces, which do not depend on the geometrical nature or the mathematical formulation of the models.

Although the basic ideas can be straightforwardly extended to geometrical multiscale scenarios, for the sake of simplicity, the target application of the present work is the weak coupling between 3D fluid flow models governed by Navier-Stokes equations. However, in Section 5.2, we briefly discuss the extension of the methodology proposed here to cover the case of a truly geometrical multiscale problem.

\subsection{Partitioning of the domain}

Let us consider a bounded domain $\Omega$ with Lipschitz continuous boundary $\partial \Omega$, which may represent a set of branching pipes. We can partition $\Omega$ into $n_{\Omega}$ subdomains $\Omega_{j}$, each one representing a specific part of the original domain. This procedure requires the imposition of continuity conditions on the fictitious boundaries $\partial \Omega_{j}^{\mathrm{CC}}$ (the interfaces), with $j=1, \ldots, n_{\Omega}$. In particular, we have

$$
\partial \Omega_{j}^{\mathrm{BC}}=\partial \Omega \cap \partial \Omega_{j}, \quad \partial \Omega_{j}^{\mathrm{CC}}=\partial \Omega_{j} \backslash \partial \Omega_{j}^{\mathrm{BC}}, \quad \forall j=1, \ldots, n_{\Omega},
$$

and

$$
\partial \Omega_{j}^{\mathrm{CC}}=\bigcup_{f=1}^{n_{\Gamma_{j}}} \Gamma_{j, f},
$$

with $n_{\Gamma_{j}}$ denoting the number of additional boundary faces $\Gamma_{j, f}$ generated by the partitioning procedure on the $j$-subdomain (see Figure 1). In the following, we refer to the continuity conditions on these faces as coupling conditions. 


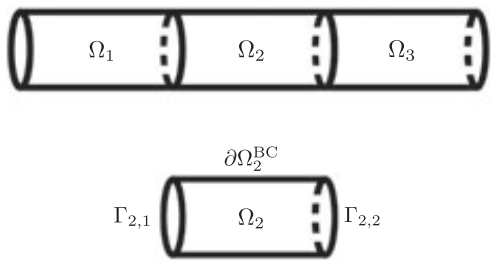

Figure 1. Decomposition of a pipe into three parts: subdomain $\Omega_{2}$ is bounded by $\partial \Omega_{2}^{\mathrm{BC}}$ plus two additional fictitious boundaries $\Gamma_{2,1}$ and $\Gamma_{2,2}$, such that $\partial \Omega_{2}^{\mathrm{CC}}=\Gamma_{2,1} \cup \Gamma_{2,2}$.

\subsection{Geometrical considerations}

In a very general setting, like the geometrical multiscale approach addressed in the introduction (see $[1,3,9]$ ), different fluid flow models can be adopted in different subdomains. Consequently, different kinds of coupling strategies could be envisaged across subdomain boundaries. From the geometrical point of view, we have two different scenarios:

1. The models are defined in the same geometrical space: coupling conditions consist in imposing directly the pointwise continuity of the unknowns seen as elements of a functional space (e.g. pointwise velocity and normal stresses for the 3D Navier-Stokes equations), which is the classical approach in domain decomposition.

2. The models are defined in different geometrical spaces: coupling conditions consist in the conservation of averaged/integrated quantities, which do not depend on the geometrical dimension of the models; that is, these are real numbers.

From the geometrical multiscale viewpoint, the main drawback of the first case is that the coupling conditions depend on the geometrical space of the models. This prevents the development of a geometrically independent strategy for the coupling of the models. However, as we show in the following, the second case can be easily applied to any geometrical scenario, even in the case of the coupling of 3D homogeneous models. In particular, in Section 4, we provide some examples of application to geometrically homogeneous 3D models. In addition, in Section 5, we briefly discuss some possible applications of the present approach to problems arising in the first scenario.

\section{Remark 1}

We underline that using the second approach the resulting model is not equivalent to the original global model. In particular, with respect to classic domain decomposition methods, the coupling conditions are relaxed by averaging/integrating the quantities over the interfaces. However, this choice allows the coupling of more than two models at the same coupling node, which is an additional appealing feature in the presence of branching elements.

\subsection{A 3D fluid flow model}

As said before, the coupling strategies developed in the forthcoming sections are general and can be employed with different physical and geometrical models. However, from now on, we focus on the 3D fluid flow modelled by the Navier-Stokes equations:

$$
\begin{cases}\rho\left(\frac{\partial \boldsymbol{u}}{\partial t}+(\boldsymbol{u} \cdot \nabla) \boldsymbol{u}\right)=-\nabla p+\nabla \cdot(2 \mu \epsilon(\boldsymbol{u}))+\boldsymbol{f} & \text { in } \Omega_{j} \times(0, T], \\ \nabla \cdot \boldsymbol{u}=0 & \text { in } \Omega_{j} \times(0, T], \\ \boldsymbol{u}=\boldsymbol{u}_{0} & \text { in } \Omega_{j} \times\{0\}, \\ \boldsymbol{u}=\mathbf{0} & \text { on } \partial \Omega_{j}^{\mathrm{BC}} \times(0, T], \\ (\boldsymbol{\sigma} \cdot \boldsymbol{n}) \cdot \boldsymbol{\tau}_{1}=\mathbf{0}, \quad(\boldsymbol{\sigma} \cdot \boldsymbol{n}) \cdot \boldsymbol{\tau}_{2}=\mathbf{0} & \text { on } \partial \Omega_{j}^{\mathrm{CC}} \times(0, T], \\ + \text { coupling condition(s) } & \text { on } \partial \Omega_{j}^{\mathrm{CC}} \times(0, T],\end{cases}
$$


where $\Omega_{j} \subset \mathbb{R}^{3}$ is the fluid domain, $(0, T]$ is the time interval, $\boldsymbol{u}$ is the velocity vector, $p$ is the hydrostatic pressure, $\mu$ is the dynamic viscosity, $\rho$ is the density, $\boldsymbol{\epsilon}(\boldsymbol{u})=\left(\boldsymbol{\nabla} \boldsymbol{u}+(\boldsymbol{\nabla u})^{T}\right) / 2$ is the strain rate tensor, $\boldsymbol{\sigma}=-p \boldsymbol{I}+2 \mu \epsilon(\boldsymbol{u})$ is the Cauchy stress tensor (with $\boldsymbol{I}$ as the identity), $\left(\boldsymbol{n}, \boldsymbol{\tau}_{1}, \boldsymbol{\tau}_{2}\right)$ are the normal and tangential directions, and $f$ represents body forces.

\section{Remark 2}

From the modelling viewpoint, we can replace $(\boldsymbol{\sigma} \cdot \boldsymbol{n}) \cdot \boldsymbol{\tau}=0$ on $\partial \Omega_{j}^{\mathrm{CC}}$ by $\boldsymbol{u} \cdot \boldsymbol{\tau}=0$. This choice is compatible with the imposition of the continuity of the normal stress and of the flow rate, described in Equation (3).

\subsection{Coupling quantities}

Under the hypothesis that all the coupling interfaces are flat and equipped with the outgoing normal $\boldsymbol{n}$, we consider, for a generic coupling interface $\Gamma$, the following choice for the coupling quantities of the fluid flow problem

$$
Q=\int_{\Gamma} \boldsymbol{u} \cdot \boldsymbol{n} \mathrm{d} \Gamma \quad \text { and } \quad \Sigma=\frac{1}{|\Gamma|} \int_{\Gamma}(\boldsymbol{\sigma} \cdot \boldsymbol{n}) \cdot \boldsymbol{n} \mathrm{d} \Gamma,
$$

where $Q$ is the volumetric flow rate and $\Sigma$ is the average of the normal component of the traction vector, hereafter referred to as the coupling stress. This choice leads to the following equations for the problem at the coupling interfaces:

$$
\forall c=1, \ldots, \mathcal{C}:\left\{\begin{array}{l}
\sum_{m=1}^{\mathcal{M}_{c}} Q_{c, m}=0, \\
\Sigma_{c, 1}=\Sigma_{c, m}, \quad \forall m=2, \ldots, \mathcal{M}_{c},
\end{array}\right.
$$

where $\mathcal{C}$ is the total number of coupling interfaces in the general framework and $\mathcal{M}_{c}$ is the number of models coupled by the $c$ th coupling interface (see Figure 2). A similar set of coupling equations is introduced in [20] for the coupling of 1D models through the conservation of the flow rate and the continuity of the total pressure.

Note that from the physical point of view, it is not realistic to introduce the partitioning in 3D simulations, unless the flow is fully developed and the section is orthogonal to the flow. However, our methodology is intended for the coupling of models of different dimensions, where the partitioning is already in the modelling.

From the practical viewpoint, on the one hand, the flow rates are imposed on problem (1) as defective boundary conditions, through Lagrange multipliers. On the other hand, the coupling stress is imposed pointwisel that is, we assume that the normal component of the traction vector is uniform on the boundaries.

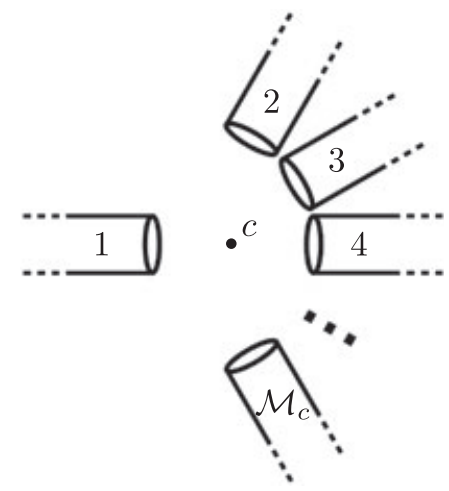

Figure 2. General configuration for the $c$ th coupling between $\mathcal{M}_{c}$ models. 


\subsection{Coupling strategies}

To satisfy the set of equations in Equation (3), we can use different coupling strategies, corresponding to the imposition of different quantities on the boundaries. In other words, we can set up each subproblem with different combinations of boundary data over the coupling interfaces. Let us introduce the strategies that we employed in the present work for setting such conditions.

Strategy $\mathcal{A}$ : The first strategy is obtained by imposing the flow rate boundary data on model 1 and the coupling stress boundary data on models $2, \ldots, \mathcal{M}_{c}$ (see Figure 2). This can be done by rearranging the equations in the following form

$$
\forall c=1, \ldots, \mathcal{C}:\left\{\begin{array}{l}
Q_{c, 1}=-\sum_{m=2}^{\mathcal{M}_{c}} Q_{c, m}\left(\Sigma_{c, m}\right), \\
\Sigma_{c, m}=\Sigma_{c, 1}\left(Q_{c, 1}\right), \quad \forall m=2, \ldots, \mathcal{M}_{c}
\end{array}\right.
$$

where we have explicitly expressed the dependence of $Q_{c, m}$ on $\Sigma_{c, m}$, and of $\Sigma_{c, 1}$ on $Q_{c, 1}$.

Thanks to Equation (4) $2, \Sigma_{c, 2}=\Sigma_{c, 3}=\cdots=\Sigma_{c, \mathcal{M}_{c}}$ and the problem can be simplified as

$$
\forall c=1, \ldots, \mathcal{C}:\left\{\begin{array}{l}
Q_{c}=-\sum_{m=2}^{\mathcal{M}_{c}} Q_{c, m}\left(\Sigma_{c}\right) \\
\Sigma_{c}=\Sigma_{c, 1}\left(Q_{c}\right)
\end{array}\right.
$$

where we highlight the presence of two coupling quantities: $Q_{c}$ and $\Sigma_{c}$. Moreover, the number of coupling equations is independent of $\mathcal{M}_{c}$. Finally, we can write the residual formulation

$$
\forall c=1, \ldots, \mathcal{C}: \quad \mathcal{R}_{c}\left(Q_{c}, \Sigma_{c}\right)=0,
$$

where

$$
\mathcal{R}_{c}=\left(\begin{array}{ccc}
-\sum_{m=2}^{\mathcal{M}_{c}} Q_{c, m}\left(\Sigma_{c}\right) & - & Q_{c} \\
\Sigma_{c, 1}\left(Q_{c}\right) & - & \Sigma_{c}
\end{array}\right)
$$

Strategy $\mathcal{B}$ : A second coupling strategy is devised by imposing a coupling stress boundary condition on all models. The residual formulation is

$$
\forall c=1, \ldots, \mathcal{C}: \quad \mathcal{R}_{c}\left(\Sigma_{c}, Q_{c, 2}, \ldots, Q_{c, \mathcal{M}_{c}}\right)=0,
$$

where

$$
\mathcal{R}_{c}=\left(\begin{array}{ccc}
Q_{c, 1}\left(\Sigma_{c}\right) & + & \sum_{m=2}^{\mathcal{M}_{c}} Q_{c, m} \\
Q_{c, 2}\left(\Sigma_{c}\right) & - & Q_{c, 2} \\
Q_{c, 3}\left(\Sigma_{c}\right) & - & Q_{c, 3} \\
& \vdots & \\
& & \\
Q_{c, \mathcal{M}_{c}}\left(\Sigma_{c}\right) & - & Q_{c, \mathcal{M}_{c}}
\end{array}\right)
$$

In this case, the number of coupling equations grows as a linear function of $\mathcal{M}_{c}$; in particular, for $\mathcal{M}_{c}>2$, the number of coupling equations is greater than the one of the previous case. 
Strategy $\mathcal{C}$ : Finally, a third coupling strategy can be devised by imposing a flow rate boundary condition on all models. The residual formulation is

$$
\forall c=1, \ldots, \mathcal{C}: \quad \mathcal{R}_{c}\left(Q_{c, 1}, Q_{c, 2}, \ldots, Q_{c, \mathcal{M}_{c}}\right)=0,
$$

where

$$
\mathcal{R}_{c}=\left(\begin{array}{ccc} 
& \sum_{m=1}^{\mathcal{M}_{c}} Q_{c, m} & \\
\Sigma_{c, 1}\left(Q_{c, 1}\right) & - & \Sigma_{c, 2}\left(Q_{c, 2}\right) \\
\Sigma_{c, 1}\left(Q_{c, 1}\right) & - & \Sigma_{c, 3}\left(Q_{c, 3}\right) \\
& \vdots & \\
\Sigma_{c, 1}\left(Q_{c, 1}\right) & - & \Sigma_{c, \mathcal{M}_{c}}\left(Q_{c, \mathcal{M}_{c}}\right)
\end{array}\right)
$$

Note that as for strategy $\mathcal{B}$, the number of coupling equations grows as a linear function of $\mathcal{M}_{c}$.

Even if strategies $\mathcal{A}, \mathcal{B}$ and $\mathcal{C}$ involve different coupling conditions, they are equivalent as they are derived from the same set of equations and coupling conditions, without any approximation. Therefore, the three systems will deliver the same solution. Nevertheless, in case of rigid models, such as the Navier-Stokes equations described in Section 2.3, strategies $\mathcal{A}$ and $\mathcal{C}$ cannot be used systematically. In fact, for the well-posedness of the subproblems, the imposition of the flow rate on all boundaries must be avoided. For strategy $\mathcal{A}$, this is always possible by carefully positioning the flow rate boundary conditions in the network. Strategy $\mathcal{C}$ can be employed only in few specific cases (e.g. the coupling between two rigid pipes, with Neumann boundary conditions on the very inflow and outflow).

\section{Remark 3}

For the sake of simplicity, in Equations (5), (6) and (7), we put in evidence only the dependence on quantities defined at the $c$ th coupling interface. Nevertheless, in a general network, boundary data at the $c$ th coupling could depend also on quantities defined at other interfaces.

\section{NUMERICAL APPROACHES}

To solve the problem described in the previous section in a segregated manner, we can use different iterative techniques. Let $\lambda=\left\{\lambda_{1}, \lambda_{2}, \ldots, \lambda_{\mathcal{C}}\right\}$ be the vector containing the coupling variables. For the strategies seen in the previous section, this becomes either

$$
\lambda_{c}^{\mathcal{A}}=\left(\begin{array}{c}
Q_{c} \\
\Sigma_{c}
\end{array}\right) \quad \text { or } \lambda_{c}^{\mathcal{B}}=\left(\begin{array}{c}
\Sigma_{c} \\
Q_{c, 2} \\
\vdots \\
Q_{c, \mathcal{M}_{c}}
\end{array}\right) \quad \text { or } \lambda_{c}^{\mathcal{C}}=\left(\begin{array}{c}
Q_{c, 1} \\
Q_{c, 2} \\
\vdots \\
\\
Q_{c, \mathcal{M}_{c}}
\end{array}\right) \forall c=1, \ldots, \mathcal{C},
$$

where we use $\mathcal{A}, \mathcal{B}$ and $\mathcal{C}$ to refer to strategies (5), (6) and (7), respectively.

\section{Remark 4}

The global vector of coupling variables $\lambda$ may consist in a combination of different types of local coupling strategies, leading to a quite arbitrary assignments of the boundary conditions on the subdomains. Such flexibility, which is another appealing feature of the present approach, holds as long as the local problems are well-posed; for instance, we cannot impose flow rate boundary conditions on all the boundaries $\Gamma_{j, f}, f=1, \ldots, n_{\Gamma_{j}}$ of the same subdomain $\Omega_{j}, j=1, \ldots, n_{\Omega}$. 


\subsection{Aitken method}

The size and nature of the problem suggest to start with a cheap method. A pure fixed-point algorithm cannot be employed because the convergence is not guaranteed. The first method that we propose is generalized Aitken's (see [21,22]), based on the following update procedure

$$
\lambda^{k+1}=\lambda^{k}+\omega^{k} \mathcal{R}\left(\lambda^{k}\right),
$$

where the value of the relaxation parameter $\omega^{k}$ is computed using one of the following formulae

$$
\begin{aligned}
& \text { Direct relaxation: } \omega^{k}=\frac{\left(\mathcal{R}\left(\lambda^{k}\right)-\mathcal{R}\left(\lambda^{k-1}\right)\right) \cdot\left(\lambda^{k}-\lambda^{k-1}\right)}{\left.\| \mathcal{R}\left(\lambda^{k}\right)-\mathcal{R}\left(\lambda^{k-1}\right)\right) \|^{2}}, \\
& \text { Inverse relaxation: } \omega^{k}=\frac{\left\|\lambda^{k}-\lambda^{k-1}\right\|^{2}}{\left(\mathcal{R}\left(\lambda^{k}\right)-\mathcal{R}\left(\lambda^{k-1}\right)\right) \cdot\left(\lambda^{k}-\lambda^{k-1}\right)} .
\end{aligned}
$$

Note that if $\omega^{k}=1$, Equation (8) is a fixed-point method. Unfortunately, the results provided by the Aitken method are not satisfactory, and convergence is guaranteed only in few simple cases, with strong restrictions on the values of $\mathcal{M}_{c}$ (see Table I).

Indeed, consider the systematic coupling of 3D fluid flow models connected in series as shown in Figure 3.

We set up a stationary simulation of a Poiseuille flow with strategies $\mathcal{A}$ and $\mathcal{B}$ using Equation (8). Even in the best case (strategy $\mathcal{A}$ with inverse relaxation), the number of iterations grows quickly with the number of elements inside the serial network, as shown in Table II.

Such unsatisfactory results motivate the use of more sophisticated approaches, like the one presented in the next section.

\subsection{Newton method}

In order to devise a convergent methodology even in the most general case, we make use of the Newton method

\begin{tabular}{|c|c|c|c|}
\hline Strategy & $\omega=1$ & Direct relaxation & Inverse relaxation \\
\hline $\mathcal{A}$ & $\begin{array}{l}\text { Slow convergence only for } \\
\max _{c=1, \ldots, \mathcal{C}}\left(\mathcal{M}_{c}\right)=2\end{array}$ & Not converging & $\begin{array}{l}\text { Convergence only for } \\
\max _{c=1, \ldots, \mathcal{C}}\left(\mathcal{M}_{c}\right)=2\end{array}$ \\
\hline $\mathcal{B}$ & $\begin{array}{l}\text { Slow convergence only for } \\
\max _{c=1, \ldots, \mathcal{C}}\left(\mathcal{M}_{c}\right)=2\end{array}$ & Not converging & Not converging \\
\hline$Q=1$ & 2 & " = & $n_{\Omega} \quad: \Sigma=0$ \\
\hline
\end{tabular}

$$
\lambda^{k+1}=\lambda^{k}+\delta \lambda^{k}
$$

Table I. Qualitative convergence results of the Aitken method.

Figure 3. Serial network of $n_{\Omega}$ cylinders connected by $\mathcal{C}=n_{\Omega}-1$ couplings. On the left, we impose a unitary flow rate, whereas on the right, a no-stress Neumann boundary condition is applied.

Table II. Convergence results of the Aitken method, for a series of elements connected one by one, such that $\max _{c=1, \ldots, \mathcal{C}}\left(\mathcal{M}_{c}\right)=2$, as shown in Figure 3.

\begin{tabular}{lccccc}
\hline $\mathcal{C}$ & 2 & 3 & 4 & 5 & 6 \\
\hline Iterations & 4 & 17 & 21 & 24 & 26 \\
\hline
\end{tabular}


where $\delta \lambda^{k}$ is computed solving the following linear system

$$
\mathcal{J}\left(\lambda^{k}\right) \delta \lambda^{k}=-\mathcal{R}\left(\lambda^{k}\right),
$$

which requires the computation of the Jacobian matrix $\mathcal{J}\left(\lambda^{k}\right)$. A general detailed description of that matrix is not available, as it depends on the structure of the graph that represents the network of couplings of the specific problem. Nevertheless, each sub-block of the matrix depends only on the type of coupling strategy, as we show in the following section.

This approach is more robust than the Aitken method: the solution is reached in only one iteration in case of the linearized Navier-Stokes equations, whereas in the non-linear case, the flow regime and the network size may affect the number of iterations.

3.2.1. Assembling the Jacobian matrix. Let $\mathcal{J}_{c_{1} c_{2}}\left(\lambda_{c_{1}}, \lambda_{c_{2}}\right)$ be a generic sub-block of the Jacobian matrix, where $c_{1}, c_{2}=1, \ldots, \mathcal{C}$ are two coupling nodes inside the network; $\mathcal{J}_{c_{1} c_{2}}\left(\lambda_{c_{1}}, \lambda_{c_{2}}\right)$ expresses the connectivity between coupling nodes $c_{1}$ and $c_{2}$ through one of the following cases:

1. $c_{1} \equiv c_{2}$ : the perturbation $\delta \lambda_{c_{2}}$ produces a variation on the coupling quantities $\lambda_{c_{2}}$ through a block diagonal element of the Jacobian matrix ( $c_{2}$ is connected with itself).

2. $c_{1} \neq c_{2}$ and these nodes are connected through a certain model: the perturbation $\delta \lambda_{c_{2}}$ produces a variation on the coupling quantities $\lambda_{c_{1}}$ through an extra diagonal block element of the Jacobian matrix.

3. $c_{1} \neq c_{2}$ and they are not connected through any model: the perturbation $\delta \lambda_{c_{2}}$ does not affect the coupling quantities $\lambda_{c_{1}}$.

The block structure of the Jacobian matrix is

$$
\mathcal{J}(\lambda)=\left[\begin{array}{cccc}
\mathcal{J}_{11}\left(\lambda_{c_{1}}, \lambda_{c_{1}}\right) & \mathcal{J}_{12}\left(\lambda_{c_{1}}, \lambda_{c_{2}}\right) & \cdots & \mathcal{J}_{1 \mathcal{C}}\left(\lambda_{c_{1}}, \lambda_{\mathcal{C}}\right) \\
\mathcal{J}_{21}\left(\lambda_{c_{2}}, \lambda_{c_{1}}\right) & \mathcal{J}_{22}\left(\lambda_{c_{2}}, \lambda_{c_{2}}\right) & \cdots & \mathcal{J}_{2 \mathcal{C}}\left(\lambda_{c_{2}}, \lambda_{\mathcal{C}}\right) \\
\vdots & \vdots & \ddots & \vdots \\
\mathcal{J}_{\mathcal{C} 1}\left(\lambda_{\mathcal{C}}, \lambda_{c_{1}}\right) & \mathcal{J}_{\mathcal{C} 2}\left(\lambda_{\mathcal{C}}, \lambda_{c_{2}}\right) & \cdots & \mathcal{J}_{\mathcal{C} \mathcal{C}}\left(\lambda_{\mathcal{C}}, \lambda_{\mathcal{C}}\right)
\end{array}\right] .
$$

In particular, for strategies $\mathcal{A}$ and $\mathcal{B}$, the sub-blocks are computed as follows.

Strategy $\mathcal{A}$ : The block diagonal elements are

$$
\mathcal{J}_{c_{1} c_{1}}\left(\lambda^{\mathcal{A}}\right)=\left[\begin{array}{cc}
-1 & -\sum_{m=2}^{\mathcal{M}_{c_{1}}} \frac{\partial Q_{c_{1}, m}}{\partial \Sigma_{c_{1}}} \\
\frac{\partial \Sigma_{c_{1}}}{\partial Q_{c_{1}}} & -1
\end{array}\right],
$$

while the pattern of the $2 \times 2$ extra diagonal blocks depends on the type of boundary conditions imposed on $c_{1}$ and $c_{2}$. More precisely,

- $\mathcal{J}_{c_{1} c_{2}}\left(\lambda^{\mathcal{A}}\right)=\left[\begin{array}{cc}0 & 0 \\ \frac{\partial \Sigma_{c_{1}}}{\partial Q_{c_{2}}} & 0\end{array}\right]$, if a flow rate is imposed on both $c_{1}$ and $c_{2}$.

- $\mathcal{J}_{c_{1} c_{2}}\left(\lambda^{\mathcal{A}}\right)=\left[\begin{array}{cc}-\frac{\partial Q_{c_{1}}}{\partial Q_{c_{2}}} & 0 \\ 0 & 0\end{array}\right]$, if a coupling stress is imposed on $c_{1}$ and a flow rate on $c_{2}$.

- $\mathcal{J}_{c_{1} c_{2}}\left(\lambda^{\mathcal{A}}\right)=\left[\begin{array}{cc}0 & -\frac{\partial Q_{c_{1}}}{\partial \Sigma_{c_{2}}} \\ 0 & 0\end{array}\right]$, if a coupling stress is imposed on both $c_{1}$ and $c_{2}$. 
$-\mathcal{J}_{c_{1} c_{2}}\left(\lambda^{\mathcal{A}}\right)=\left[\begin{array}{cc}0 & 0 \\ & \\ \text { stress on } c_{2} \text {. } & \frac{\partial \Sigma_{c_{1}}}{\partial \Sigma_{c_{2}}}\end{array}\right]$, if a flow rate is imposed on $c_{1}$ and a coupling

Strategy $\mathcal{B}$ : The block diagonal elements are

$$
\mathcal{J}_{c_{1} c_{1}}\left(\lambda^{\mathcal{B}}\right)=\left[\begin{array}{ccccc}
\frac{\partial Q_{c_{1}, 1}}{\partial \Sigma_{c_{1}}} & 1 & 1 & \cdots & 1 \\
\frac{\partial Q_{c_{1}, 2}}{\partial \Sigma_{c_{1}}} & -1 & 0 & \cdots & 0 \\
\frac{\partial Q_{c_{1}, 3}}{\partial \Sigma_{c_{1}}} & 0 & -1 & & \vdots \\
\vdots & \vdots & & \ddots & \vdots \\
\frac{\partial Q_{c_{1}, \mathcal{M}_{1}}}{\partial \Sigma_{c_{1}}} & 0 & \cdots & \cdots & -1
\end{array}\right]
$$

whereas all the extra diagonal blocks assume the following form

$$
\mathcal{J}_{c_{1} c_{2}}\left(\lambda^{\mathcal{B}}\right)=\left[\begin{array}{cccc}
0 & 0 & \cdots & 0 \\
\vdots & \vdots & & \vdots \\
0 & 0 & \cdots & 0 \\
\frac{\partial Q_{c_{1}, v_{1}}}{\partial \Sigma_{c_{2}}} & 0 & \cdots & 0 \\
0 & 0 & \cdots & 0 \\
\vdots & \vdots & & \vdots \\
0 & 0 & \cdots & 0
\end{array}\right],
$$

where $1 \leq v_{c_{1}} \leq \mathcal{V}_{c_{1}}$, with $v_{c_{1}}$ is the index of the corresponding coupling interface at $c_{1}$ and $\mathcal{V}_{c_{1}}$ is the local number of coupling variables at $c_{1}$, that is, the length of $\lambda_{c_{1}}$. The size of the blocks of the matrix is $\mathcal{M}_{c_{1}} \times \mathcal{M}_{c_{1}}$ for the diagonal elements and $\mathcal{M}_{c_{1}} \times \mathcal{M}_{c_{2}}$ for the extra diagonal ones.

Strategy $\mathcal{C}:$ The block diagonal elements are

$$
\mathcal{J}_{c_{1} c_{1}}\left(\lambda^{\mathcal{C}}\right)=\left[\begin{array}{ccccc}
1 & 1 & 1 & \cdots & 1 \\
\frac{\partial \Sigma_{c_{1}, 1}}{\partial Q_{c_{1}, 1}} & -\frac{\partial \Sigma_{c_{1}, 2}}{\partial Q_{c_{1}, 2}} & 0 & \cdots & 0 \\
\frac{\partial \Sigma_{c_{1}, 1}}{\partial Q_{c_{1}, 1}} & 0 & -\frac{\partial \Sigma_{c_{1}, 3}}{\partial Q_{c_{1}, 3}} & & \vdots \\
\vdots & \vdots & & \ddots & \vdots \\
\frac{\partial \Sigma_{c_{1}, 1}}{\partial Q_{c_{1}, 1}} & 0 & \cdots & \cdots & -\frac{\partial \Sigma_{c_{1}, \mathcal{M}_{1}}}{\partial Q_{c_{1}, \mathcal{M}_{1}}}
\end{array}\right],
$$

whereas all the extra diagonal blocks, according to the interface equation, either become 


$$
\begin{aligned}
& \mathcal{J}_{c_{1} c_{2}}\left(\lambda^{\mathcal{C}}\right)=\left[\begin{array}{ccccccc}
0 & \cdots & 0 & 0 & 0 & \cdots & 0 \\
0 & \cdots & 0 & \frac{\partial \Sigma_{c_{1}, 1}}{\partial Q_{c_{2}, v_{2}}} & 0 & \cdots & 0 \\
0 & \cdots & 0 & \frac{\partial \Sigma_{c_{1}, 1}}{\partial Q_{c_{2}, v_{2}}} & 0 & \cdots & 0 \\
\vdots & & \vdots & \vdots & \vdots & & \vdots \\
0 & \cdots & 0 & \frac{\partial \Sigma_{c_{1}, 1}}{\partial Q_{c_{2}, v_{c_{2}}}} & 0 & \cdots & 0
\end{array}\right], \quad \text { or } \\
& \mathcal{J}_{c_{1} c_{2}}\left(\lambda^{\mathcal{C}}\right)=\left[\begin{array}{ccccccc}
0 & \cdots & 0 & 0 & 0 & \cdots & 0 \\
\vdots & & \vdots & \vdots & \vdots & & \vdots \\
0 & \cdots & 0 & 0 & 0 & \cdots & 0 \\
0 & \cdots & 0 & -\frac{\partial \Sigma_{c_{1}, v_{1}}}{\partial Q_{c_{2}, v_{c_{2}}}} & 0 & \cdots & 0 \\
0 & \cdots & 0 & 0 & 0 & \cdots & 0 \\
\vdots & & \vdots & \vdots & \vdots & & \vdots \\
0 & \cdots & 0 & 0 & 0 & \cdots & 0
\end{array}\right]
\end{aligned}
$$

where $1 \leq v_{c_{2}} \leq \mathcal{V}_{c_{2}}$, with $v_{c_{2}}$ the index of the corresponding coupling interface at $c_{2}$ and $\mathcal{V}_{c_{2}}$ the local number of coupling variables at $c_{2}$, i.e., the length of $\lambda_{c_{2}}$. As for strategy $\mathcal{B}$, the size of the blocks of the matrix is $\mathcal{M}_{c_{1}} \times \mathcal{M}_{c_{1}}$ for the diagonal elements and $\mathcal{M}_{c_{1}} \times \mathcal{M}_{c_{2}}$ for the extra diagonal ones.

Note that in some particular cases, for example, $c_{1}$ and $c_{2}$ being connected by a rigid pipe, some derivatives may be trivial: $\partial Q_{c_{1}} / \partial Q_{c_{2}}=-1$.

To assemble the Jacobian matrix (10), we can use different approaches. An instance is provided in Algorithm 1, which builds the Jacobian by blocks.

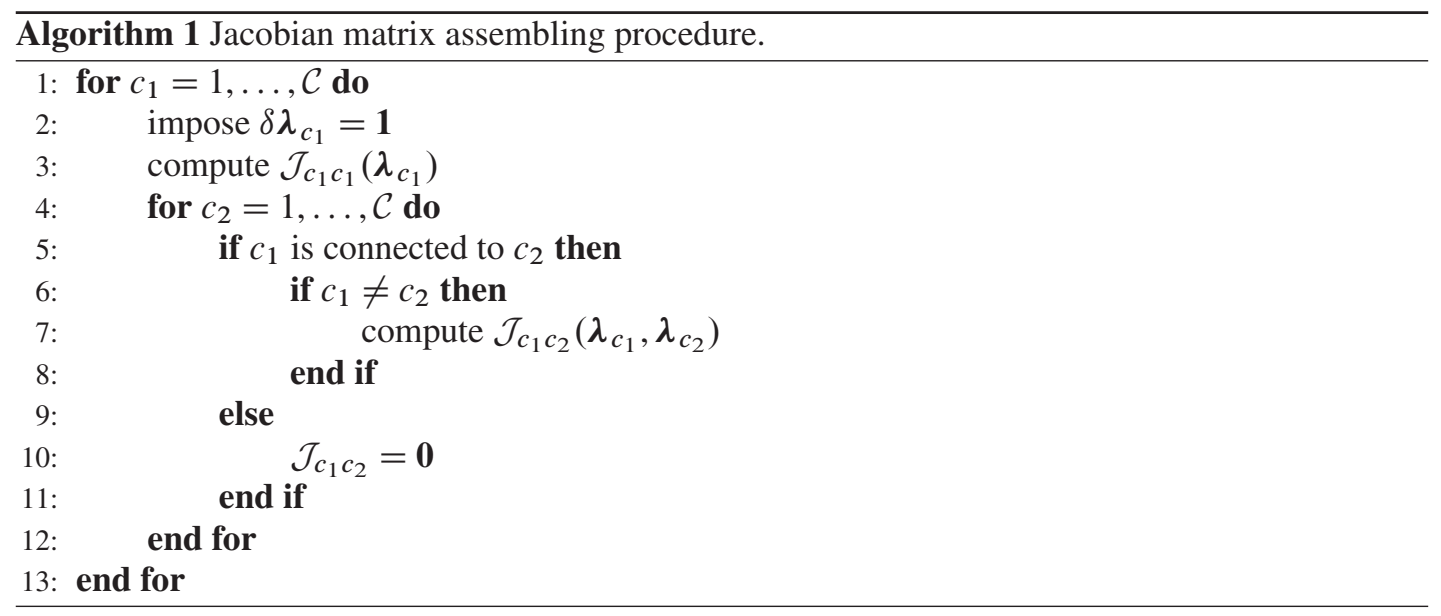

A practical example of the resulting matrices is presented in Section 4.1, whereas the computation of the Jacobian entries is outlined in the following section. 
3.2.2. Computation of the Jacobian coefficients. The computation of the coefficients of the Jacobian matrix depends on the equations used within each subdomain. In $[14,15]$, the authors use a finite difference approximation to estimate the coefficients. The main drawback of this approach is that the accuracy of the approximation depends on the choice of the perturbation magnitude to be applied to the coupling quantity, and therefore, it may have a strong impact on the global number of iterations required by the Newton scheme. In the present work, we provide an explicit expression of the Jacobian coefficient of the 3D Navier-Stokes equations in Equation (1), by solving the tangent problem formulated on the subdomain $\Omega_{j}$ (see [23]). With this approach, the magnitude of the perturbation does not have any impact on the accuracy of the result. Let us introduce $\mathcal{L}_{j}^{Q}$ and $\mathcal{L}^{\Sigma_{j}}$ as the lists of boundaries of $\Omega_{j}$ where flow rate and coupling stress conditions are applied, respectively. Given $\boldsymbol{u} \in \mathcal{U}\left(\Omega_{j}\right), \delta Q_{f}, f \in \mathcal{L}_{j}^{Q}$ and $\delta \Sigma_{f}, f \in \mathcal{L}^{\Sigma_{j}}$, find $\delta \boldsymbol{u} \in \mathcal{U}\left(\Omega_{j}\right), \delta p \in L^{2}\left(\Omega_{j}\right)$ and $\delta \Lambda_{f} \in \mathbb{R}, f \in \mathcal{L}_{j}^{Q}$, such that

$$
\left\{\begin{array}{lr}
\int_{\Omega_{j}} \rho \frac{\partial \delta \boldsymbol{u}}{\partial t} \cdot \boldsymbol{v} \mathrm{d} \Omega+\int_{\Omega_{j}} \rho(\delta \boldsymbol{u} \cdot \nabla) \boldsymbol{u} \cdot \boldsymbol{v} \mathrm{d} \Omega+\int_{\Omega_{j}} \rho(\boldsymbol{u} \cdot \nabla) \delta \boldsymbol{u} \cdot \boldsymbol{v} \mathrm{d} \Omega-\int_{\Omega_{j}} \delta p \nabla \cdot \boldsymbol{v} \mathrm{d} \Omega & \\
+\int_{\Omega_{j}} \mu \nabla \delta \boldsymbol{u} \cdot \nabla \boldsymbol{v} \mathrm{d} \Omega+\sum_{f \in \mathcal{L}_{j}^{Q}} \int_{\Gamma_{j, f}} \delta \Lambda_{f} \boldsymbol{v} \cdot \boldsymbol{n} \mathrm{d} \Gamma=-\sum_{f \in \mathcal{L}^{\Sigma_{j}}} \int_{\Gamma_{j, f}} \delta \Sigma_{f} \boldsymbol{v} \cdot \boldsymbol{n} \mathrm{d} \Gamma & \forall \boldsymbol{v} \in \mathcal{V}\left(\Omega_{j}\right), \\
\int_{\Omega_{j}} q \nabla \cdot \delta \boldsymbol{u} \mathrm{d} \Omega=0 & \forall q \in L^{2}\left(\Omega_{j}\right), \\
\int_{\Gamma_{j, f}} \delta \boldsymbol{u} \cdot \boldsymbol{n} \mathrm{d} \Gamma=\delta Q_{f} & f \in \mathcal{L}_{j}^{Q},
\end{array}\right.
$$

where in $\mathcal{U}\left(\Omega_{j}\right)$, we consider the essential boundary conditions as given in Equation (1) and $\mathcal{V}\left(\Omega_{j}\right)$ is the associated linear space. With this set of equations, we compute the coefficients of the block matrices $\mathcal{J}_{c_{1}, c_{2}}$, with $c_{1}, c_{2}=1, \ldots, \mathcal{C}$, that are used in the assembling procedure of the Jacobian matrix. First of all, we have to find which are the boundaries $\Gamma_{j, f_{1}}$ and $\Gamma_{j, f_{2}}$ associated with the couplings $c_{1}$ and $c_{2}$, respectively. Then, we compute the coefficients in the following way:

- $\frac{\partial Q_{c_{1}}}{\partial Q_{c_{2}}}$ and $\frac{\partial \Sigma_{c_{1}}}{\partial Q_{c_{2}}}$ are computed as the resulting flow rate and coupling stress from the imposition to Equation (11), of a variation $\delta Q_{c_{2}}=1$ on $\Gamma_{j, f_{2}}$ and homogeneous boundary conditions of the corresponding type on all the other boundaries, that is, $\delta Q_{f}=0, \forall f \in \mathcal{L}_{j}^{Q}, f \neq f_{2}$ and $\delta \Sigma_{f}=0, \forall f \in \mathcal{L}_{j}^{\Sigma}$ (see Figures $4(\mathrm{a}, \mathrm{b})$ );

- $\frac{\partial \Sigma_{c_{1}}}{\partial \Sigma_{c_{2}}}$ and $\frac{\partial Q_{c_{1}}}{\partial \Sigma_{c_{2}}}$ are computed as the resulting coupling stress and flow rate from the imposition to Equation (11) of a variation $\delta \Sigma_{c_{2}}=1$ on $\Gamma_{j, f_{2}}$ and homogeneous boundary conditions

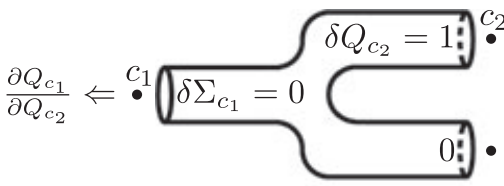

(a)

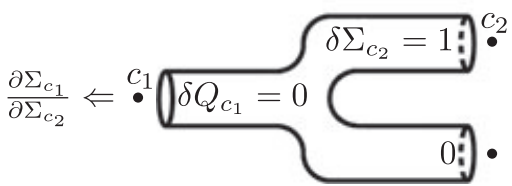

(c)

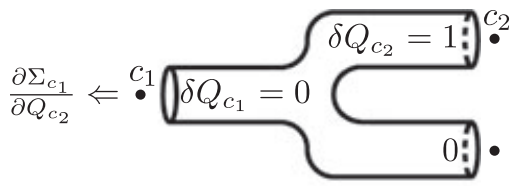

(b)

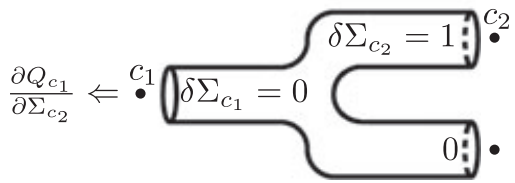

(d)

Figure 4. Example of computation of the Jacobian coefficients on a bifurcation. In the sketches, the zero on the lower branch of the bifurcation stands for a homogeneous value of the corresponding boundary condition ( $Q$ or $\Sigma$, depending on the nature of the original boundary data). (a) Computation of $\frac{\partial Q_{c_{1}}}{\partial Q_{c_{2}}}$. (b) Computation

$$
\text { of } \frac{\partial \Sigma_{c_{1}}}{\partial Q_{c_{2}}} \text {. (c) Computation of } \frac{\partial \Sigma_{c_{1}}}{\partial \Sigma_{c_{2}}} \text {. (d) Computation of } \frac{\partial Q_{c_{1}}}{\partial \Sigma_{c_{2}}} \text {. }
$$


of the corresponding type on all the other boundaries, that is, $\delta Q_{f}=0, \forall f \in \mathcal{L}_{j}^{Q}$ and $\delta \Sigma_{f}=0, \forall f \in \mathcal{L}_{j}^{\Sigma}, f \neq f_{2}$ (see Figure 4(c,d)).

\section{Remark 5}

The assembly procedure described in Algorithm 1 is quite naive and not very efficient, as in general, it leads to performing multiple perturbations of the same coupling variable $\lambda_{c, v}$ and, hence, to performing multiple solutions of the same linear system. To minimize the computational cost it is necessary to assemble the matrix column by column; in other words, for each perturbation $\delta \lambda_{c, v}$, we solve the linear system associated to each problem only once, computing all the coefficients in the same column.

\section{RESULTS}

In this section, we show some numerical results obtained using the coupling strategies and the numerical algorithms presented in the previous sections. In particular, we present three examples: the first one is a steady Stokes test case, which helps us on giving more details about the main features of the presented methods. In the second example, we make use of the coupling strategies to solve the Womersley flow (see [24]) and present a quantitative comparison between this numerical solution and the exact velocity profile. Finally, in the third case, we simulate the blood flow in the carotid bifurcation under physiological regimes. In all the examples, the Navier-Stokes equations (Equation (1)) are solved using a $\mathbb{P} 1-\mathbb{P} 1$ finite element method, stabilized through interior penalty (see [25]), which shows a convergence of order one in $h$ (the spatial discretization of the whole domain $\Omega$ ) for both velocity and pressure. In addition, we use a first-order backward Euler scheme for the time discretization. This choice may affect the result computed in each single domain and hence the solution of the global coupled problem; however, it does not have any impact on the coupling strategies. As discussed at the end of Section 2.5, strategy $\mathcal{C}$ cannot be used systematically to couple 3D rigid wall models; for this reason, in the following, we limit our analysis to strategies $\mathcal{A}$ and $\mathcal{B}$.

\subsection{Seven cylinders in steady Stokes}

The first example that we propose is a 3D steady Stokes problem, whose domain consists in a set of seven cylinders connected by four coupling interfaces, as shown in Figure 5(a). All the cylinders have the same dimensions (radius 0.08 ; length 0.4 ). A unitary flow rate is imposed on the leftmost side, whereas a homogeneous Neumann boundary condition is applied on the outflow, on the right.

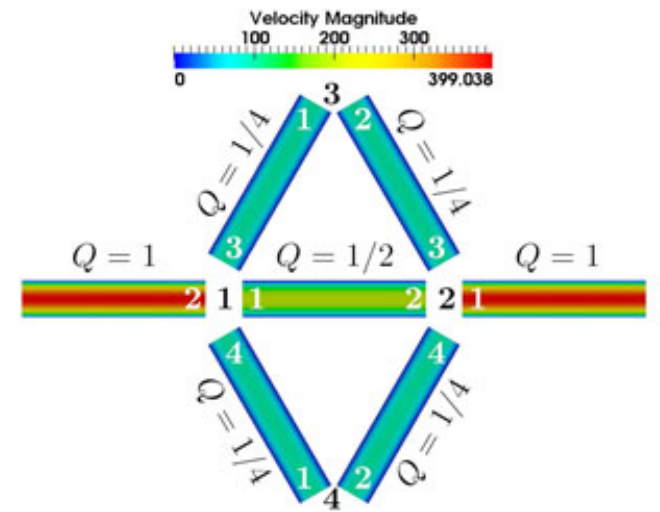

(a)

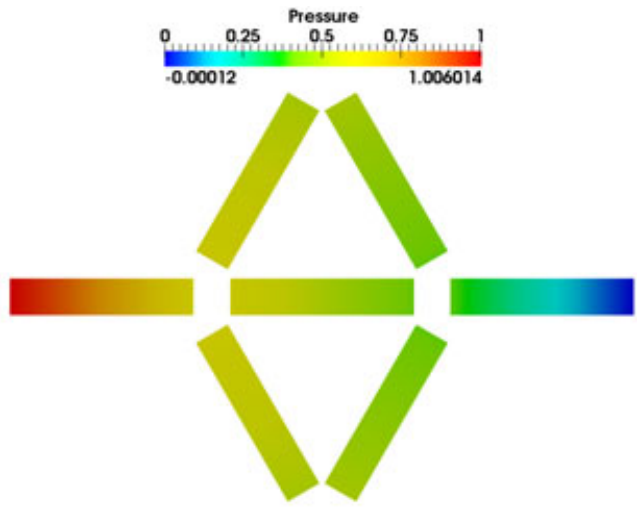

(b)

Figure 5. Configuration and solution of seven cylinders in a steady Stokes simulation. (a) Velocity magnitude; black numbers indicate the coupling interfaces, while white numbers indicate the local numeration of the boundaries. On top of the domains the analytical solution is provided. (b) Pressure field: the drop pressure between the inflow and the outflow is equal to one. The values of the analytical solution of the pressure at the four coupling points are: $2 / 3$ at $c=1,1 / 3$ at $c=2$, and $1 / 2$ at $c=3,4$. 
The physical quantities (density and viscosity) are chosen in order to obtain a unitary pressure drop along the network. The solution obtained with strategies $\mathcal{A}$ and $\mathcal{B}$ is shown in Figure 5.

As discussed at the end of Section 2.5, in presence of rigid models, strategy $\mathcal{A}$ cannot be used systematically. In particular, we have to guarantee that all the subproblems have at least one Neumann boundary condition. In the present example, this has been done as described in Figure 6.

The resulting vectors of coupling variables and the residual equations are

$\lambda^{\mathcal{A}, k}=\left(\begin{array}{c}Q_{1}^{k} \\ \Sigma_{1}^{k} \\ Q_{2}^{k} \\ \Sigma_{2}^{k} \\ Q_{3}^{k} \\ \Sigma_{3}^{k} \\ \frac{Q_{4}^{k}}{\Sigma_{4}^{k}}\end{array}\right), \quad \mathcal{R}\left(\lambda^{\mathcal{A}, k}\right)=\left(\begin{array}{lr}-Q_{1,2}\left(\Sigma_{1}^{k}\right)-Q_{1,3}\left(\Sigma_{1}^{k}, Q_{3}^{k}\right)-Q_{1,4}\left(\Sigma_{1}^{k}, Q_{4}^{k}\right) & -Q_{1}^{k} \\ \Sigma_{1,1}\left(Q_{1}^{k}, \Sigma_{2}^{k}\right) & -\Sigma_{1}^{k} \\ -Q_{2,2}\left(Q_{1}^{k}, \Sigma_{2}^{k}\right)-Q_{2,3}\left(\Sigma_{2}^{k}, \Sigma_{3}^{k}\right)-Q_{2,4}\left(\Sigma_{2}^{k}, \Sigma_{4}^{k}\right)-Q_{2}^{k} \\ \Sigma_{2,1}\left(Q_{2}^{k}\right) & -\Sigma_{2}^{k} \\ -Q_{3,2}\left(\Sigma_{2}^{k}, \Sigma_{3}^{k}\right) & -Q_{3}^{k} \\ \Sigma_{3,1}\left(\Sigma_{1}^{k}, Q_{3}^{k}\right) & -Q_{4}^{k} \\ -Q_{4,2}\left(\Sigma_{2}^{k}, \Sigma_{4}^{k}\right) & -\Sigma_{4}^{k}\end{array}\right)$,

$$
\lambda^{\mathcal{B}, k}=\left(\begin{array}{c}
\Sigma_{1}^{k} \\
Q_{1,2}^{k} \\
Q_{1,3}^{k} \\
Q_{1,4}^{k} \\
\hline \Sigma_{2}^{k} \\
Q_{2,2}^{k} \\
Q_{2,3}^{k} \\
Q_{2,4}^{k} \\
\frac{\Sigma_{3}^{k}}{Q_{3,2}^{k}} \\
\frac{\Sigma_{4}^{k}}{Q_{4,2}^{k}}
\end{array}\right), \quad \mathcal{R}\left(\lambda^{\mathcal{B}, k}\right)=\left(\begin{array}{l}
Q_{1,1}\left(\Sigma_{1}^{k}, \Sigma_{2}^{k}\right)+Q_{1,2}^{k}+Q_{1,3}^{k}+Q_{1,4}^{k} \\
Q_{1,2}\left(\Sigma_{1}^{k}\right)-Q_{1,2}^{k} \\
Q_{1,3}\left(\Sigma_{1}^{k}, \Sigma_{3}^{k}\right)-Q_{1,3}^{k} \\
Q_{1,4}\left(\Sigma_{1}^{k}, \Sigma_{4}^{k}\right)-Q_{1,4}^{k} \\
Q_{2,1}\left(\Sigma_{2}^{k}\right)+Q_{2,2}^{k}+Q_{2,3}^{k}+Q_{2,4}^{k} \\
Q_{2,2}\left(\Sigma_{1}^{k}, \Sigma_{2}^{k}\right)-Q_{2,2}^{k} \\
Q_{2,3}\left(\Sigma_{2}^{k}, \Sigma_{3}^{k}\right)-Q_{2,3}^{k} \\
Q_{2,4}\left(\Sigma_{2}^{k}, \Sigma_{4}^{k}\right)-Q_{2,4}^{k} \\
\frac{Q_{3,1}\left(\Sigma_{1}^{k}, \Sigma_{3}^{k}\right)+Q_{3,2}^{k}}{Q_{3,2}\left(\Sigma_{2}^{k}, \Sigma_{3}^{k}\right)-Q_{3,2}^{k}} \\
\frac{Q_{4,1}\left(\Sigma_{1}^{k}, \Sigma_{4}^{k}\right)+Q_{4,2}^{k}}{Q_{4,2}\left(\Sigma_{2}^{k}, \Sigma_{4}^{k}\right)-Q_{4,2}^{k}}
\end{array}\right),
$$

where the numeration follows the scheme of Figure 6. The Aitken method does not converge, because of the complex pattern of the network, whereas the Newton method converges in one 
iteration because the fluid flow problem defined inside each cylinder is linear. The Jacobian matrices for the coupling strategies are

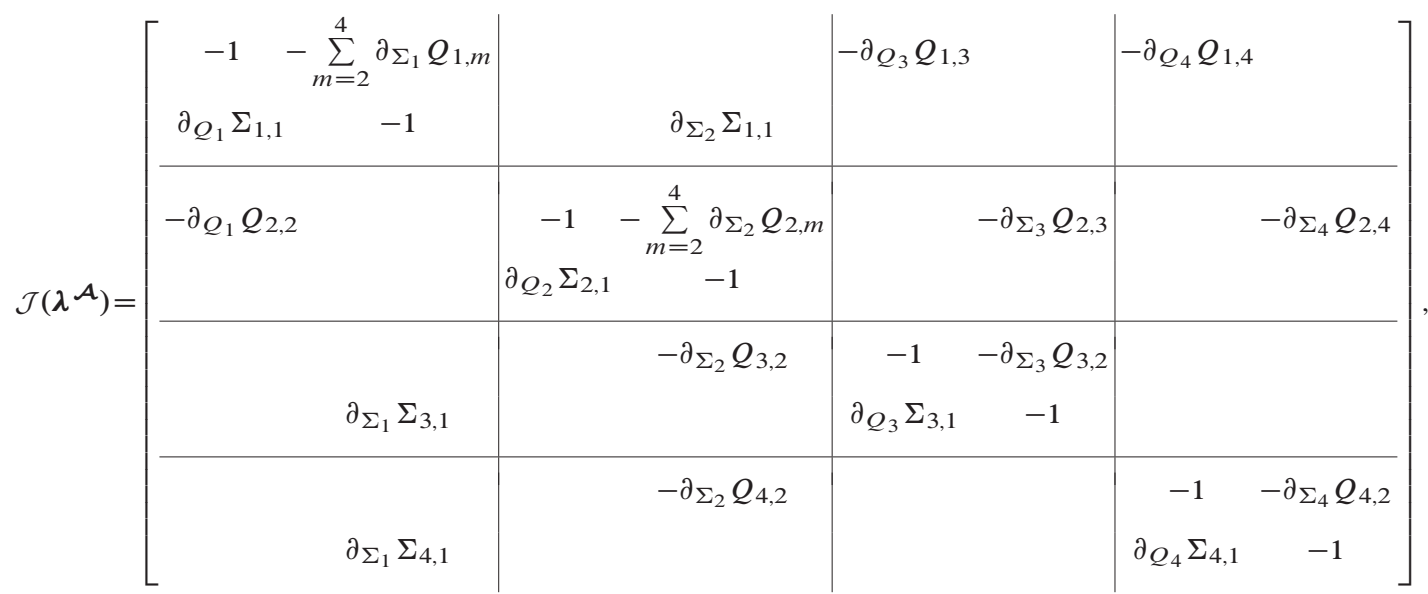

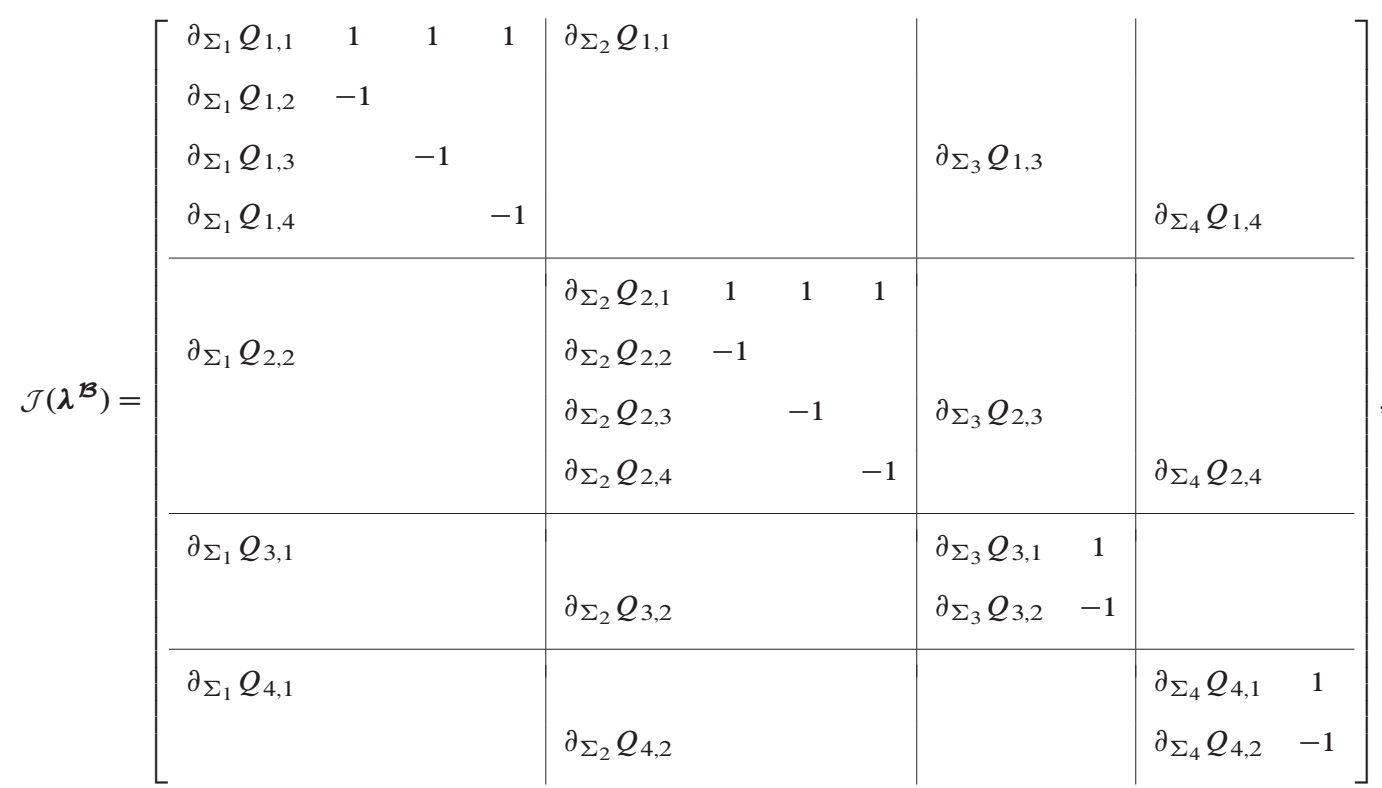

where we use the compact notation $\partial_{Q}$ and $\partial_{\Sigma}$ to express the partial derivative of a quantity, with respect to the flow rate and the coupling stress, respectively; the white spaces indicate null entries. To compute all the coefficients of the Jacobian matrix, we perform 12 evaluations of problem (11). This number is directly related to the implementation of the assembling algorithm, which in our case is optimal with respect to the number of evaluations of the linear problems associated to the Jacobian (see Remark 5). For this problem, in which we have the flow rate and the coupling stress as the unknowns, this number is equal to the number of artificial boundary surfaces created by the decomposition of the original domain $\Omega$ into subdomains $\Omega_{j}, j=1, \ldots, n_{\Omega}$ (12 in this example). Therefore, at each subiteration of the Newton method, we need to perform $\sum_{j=1}^{n_{\Omega}} n_{\Gamma_{j}}$ evaluations of problem (11), plus $n_{\Omega}$ evaluations of problem (1) to evaluate the residual. These operations can be done in a complete parallel fashion, as we will discuss in Section 5.1.

The results obtained with strategies $\mathcal{A}$ and $\mathcal{B}$ are equivalent. The computed flow rate is exact up to the imposed tolerance for the Stokes solver. The convergence of the coupling stress with respect to $h$ is shown in Figure 7. 

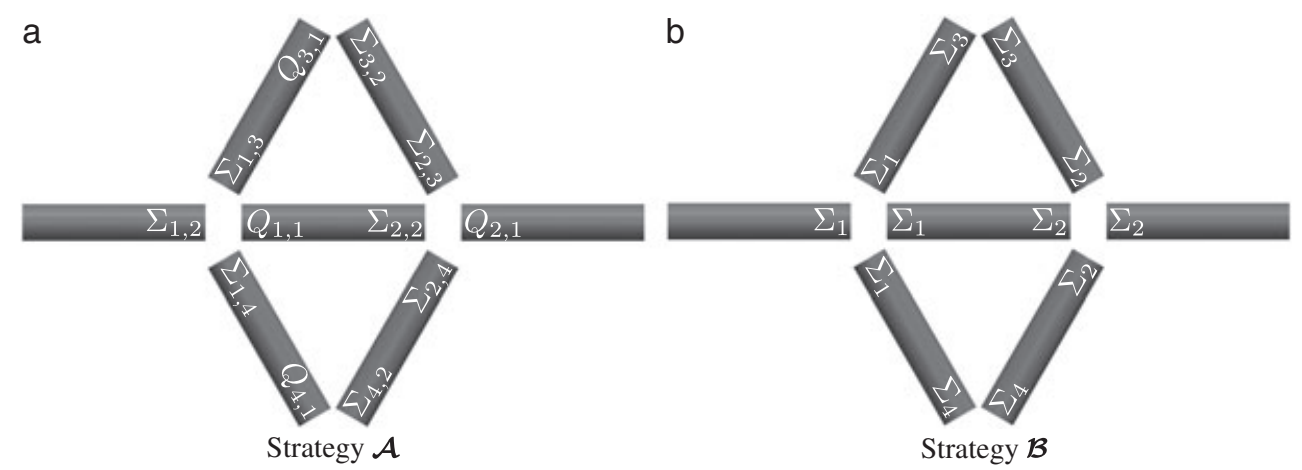

Figure 6. Coupling conditions for the example of Figure 5. (a) All the pipes have at least one Neumann boundary condition. (b) Strategy $\mathfrak{B}$ can be used systematically without any constraint.

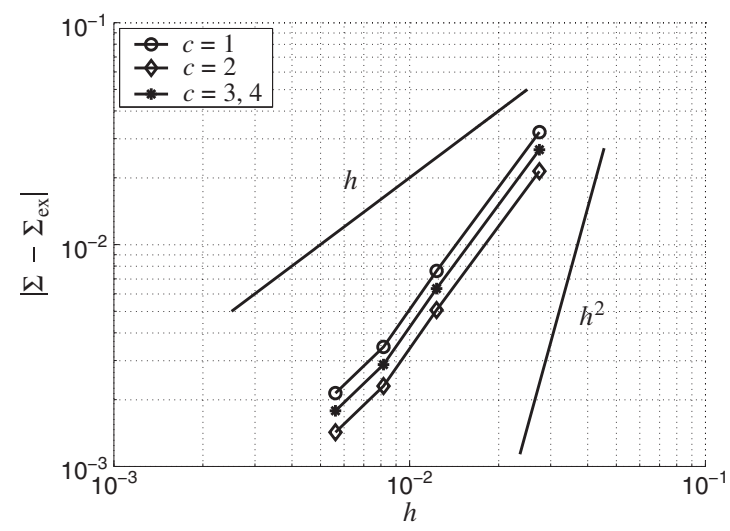

Figure 7. Normalized coupling stress error at the four coupling interfaces of the example in Figure 5(a). Strategies $\mathcal{A}$ and $\mathcal{B}$ give the same result.

\subsection{Five cylinders in a Womersley regime}

The second example that we propose is a Womersley fluid flow (see [24]) in a cylindrical domain $\Omega$ composed by five subdomains $\Omega_{j}$ of equal size and shape discretized by the same unstructured mesh (with $h / D=0.15415$, where $D$ is the diameter). On the leftmost inflow, we impose a sinusoidal flow rate $Q=-A \sin (2 \pi t / T)$, whereas a homogeneous Neumann boundary condition is applied on the rightmost outflow. The amplitude $A$, the period $T$ and all the physical quantities are chosen in order to obtain a Womersley number of 5 and a Reynolds number of 600, which are typical values in a human carotid. The time step is given by $T / 256$.

For this problem, each subiteration of the Newton method requires eight evaluations of problem (11) to assemble the Jacobian matrix, plus five evaluations of problem (1) to compute the solution in the 3D domains once the linear system (9) is solved. This approach ensures that the solution satisfies, at each time step, the continuity equations (Equation (3)) at the coupling interfaces among the five subdomains. As for the example described in Section 4.1, the solutions computed using strategies $\mathcal{A}$ and $\mathcal{B}$ are the same. Moreover, also the velocity profiles in the different subdomains coincide. In Figure 8, we show a comparison between the magnitude of the computed velocity profiles and the analytical solution, at selected times. From these graphs, we observe that the velocity profiles follow the shape of the exact solution.

\subsection{Carotid bifurcation}

In this last example, we use the methodology devised in this paper to compare the solution obtained in a physiological carotid bifurcation (PCB), with the one given by a synthetic carotid bifurcation 


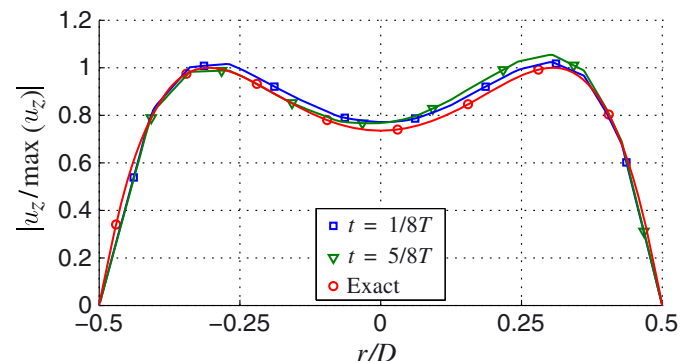

(a)

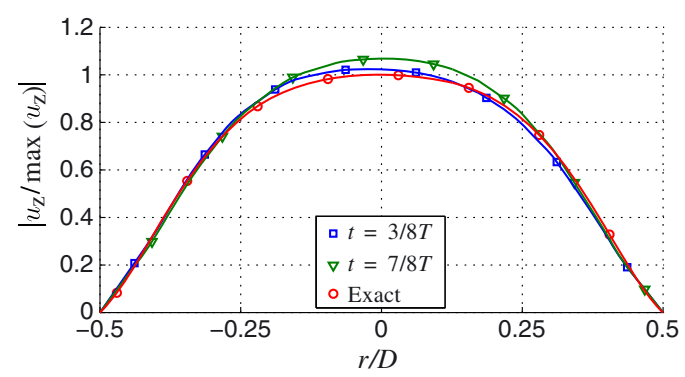

(c)

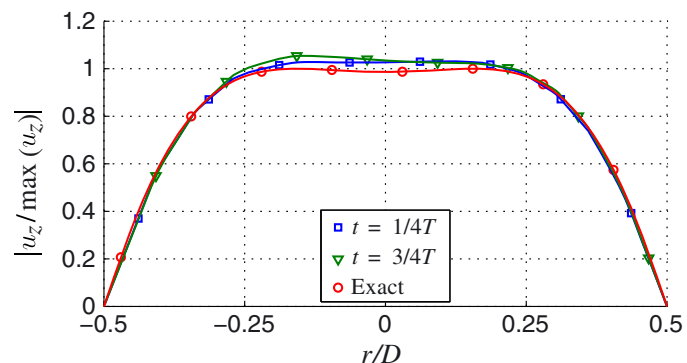

(b)

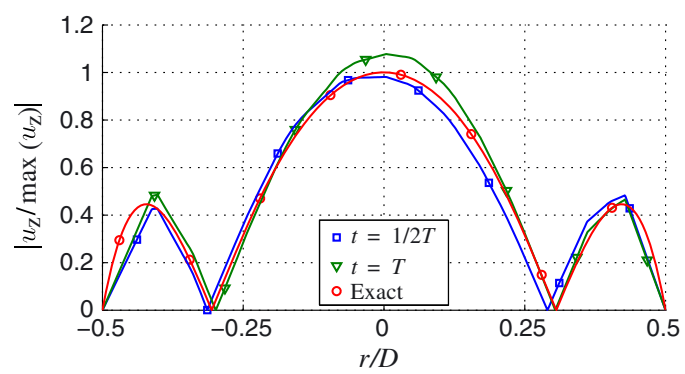

(d)

Figure 8. Womersley velocity profiles: on the $x$-axis, we show the ratio between the radial coordinate and the diameter, whereas on the $y$-axis, we display the modulus of the axial velocity, normalized on the maximum value of the exact solution.

(SCB). The geometries and the arrangements of the two schemes are described in Figure 9. It is important to point out that the goal of this example is on the application of the decomposition strategy to a problem with some physiological meaning. This kind of comparison may give insight on the differences in global quantities (flow rate and coupling stress) that should be expected when using a simple version of a bifurcation such as the SCB when compared with the realistic geometry $\mathrm{PBC}$. We are by no means interested in trying to recover the 3D features of the flow computed in the PCB with the SCB.

For the physical quantities, we use physiological values $\left(\rho=1 \mathrm{~g} / \mathrm{cm}^{3}, \mu=0.035 \mathrm{~g} / \mathrm{cm} / \mathrm{s}\right)$, imposing a heart systole-diastole flow rate cycle (corresponding to 75 beats per minute) at the inlet of $\Omega_{1}$, whereas a homogeneous Neumann boundary condition is applied on the outflow of $\Omega_{2}$ and $\Omega_{3}$. It is worth pointing out that the two configurations feature a different number of coupling interfaces. Therefore, for the PCB, each subiteration of the Newton method requires six evaluations of problem (11), plus four evaluations of problem (1), whereas for the SCB, it requires nine evaluations of problem (11), plus six evaluations of problem (1).

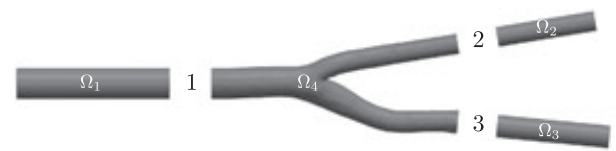

(a)

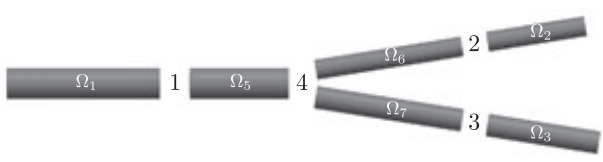

(b)

Figure 9. Carotid bifurcation schemes: subdomains $\Omega_{1}, \Omega_{2}$ and $\Omega_{3}$ are cylinders with $r / L=0.1$. In addition, they are scaled in order to have the same area of the corresponding interfaces of the physiological carotid bifurcation $\Omega_{4}$. (a) Physiological carotid: $\Omega_{4}$. The values of the inlet/outlet areas are $\left|\Gamma_{4,1}\right|=0.351 \mathrm{~cm}^{2},\left|\Gamma_{4,2}\right|=0.147 \mathrm{~cm}^{2}$ and $\left|\Gamma_{4,3}\right|=0.190 \mathrm{~cm}^{2}$. (b) Synthetic carotid composed by three cylinders: $\Omega_{5}, \Omega_{6}$ and $\Omega_{7}$. The area of the cylinders match the ones of the three interfaces of the physiological carotid $\Omega_{4}$. 
For the two configurations, we compare the flow division and the coupling stress at the different coupling interfaces. In addition, we compare also the velocity profiles inside the two carotids. In Figure 10, the evolution of the flow rate during one heart beat is shown. In particular, in Figure 10(a), the small difference (around 10\%), between the flow rates of the PCB with respect to the ones of the SCB (at $c=2$ and $c=3$ ), is due to the different approximation used at the bifurcation, which, in the case of the SCB, neglects the effects of the 3D geometry. Nevertheless, we obtain a good separation of the flows in the SCB when compared with the PCB.

Regarding the coupling stress (Figure 11), we observe that there are no significant differences between the PCB and the SCB as this quantity mainly depends on the global length of the geometrical model, rather than on the 3D geometry.

Finally, in Figure 12, we show a qualitative comparison between the velocity profiles of the two carotids, at selected times. As expected, in the SCB the velocity assumes a Womersley-like profile, which is not the case of the $\mathrm{PCB}$, where the shape of the $3 \mathrm{D}$ bifurcation produces asymmetries in the solution. Nevertheless, the magnitude of the velocity in the two cases is nearly the same (see the colours in Figure 12). In addition, we observe that the velocity profiles at the coupling interfaces agree well in both cases.

\section{EXTENSIONS AND APPLICATIONS}

In this section, we comment about the implementation of our methodology in a parallel framework; then, we address two other possible fields of application, where the main ideas proposed in this paper can be effectively used.

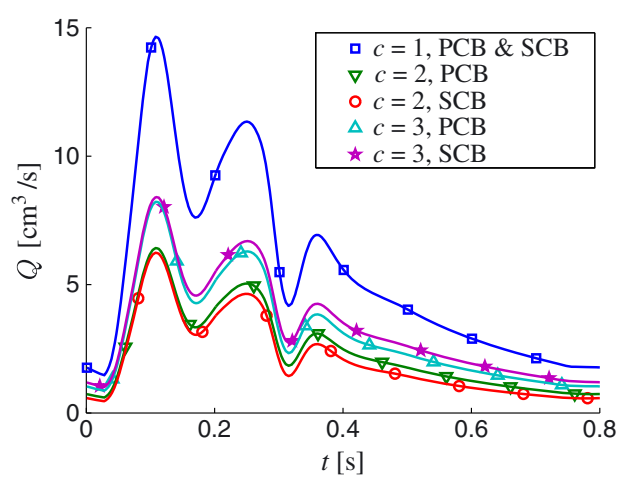

(a)

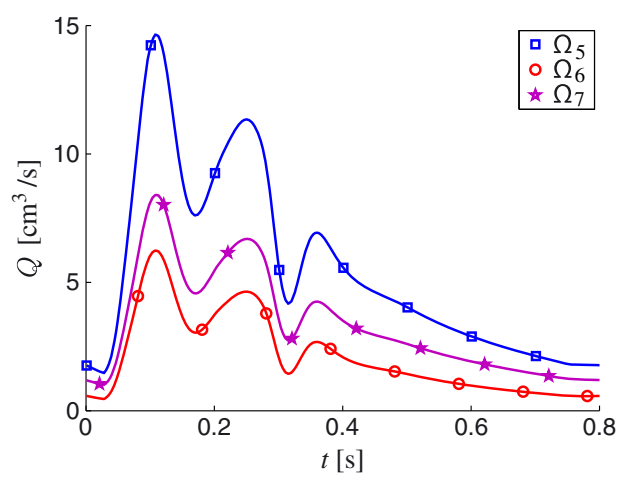

(b)

Figure 10. (a) Flow rate at the different coupling interfaces of the physiological carotid bifurcation (PCB) and the synthetic carotid bifurcation (SCB). (b) Flow rate splitting at $c=4$ of the SCB.

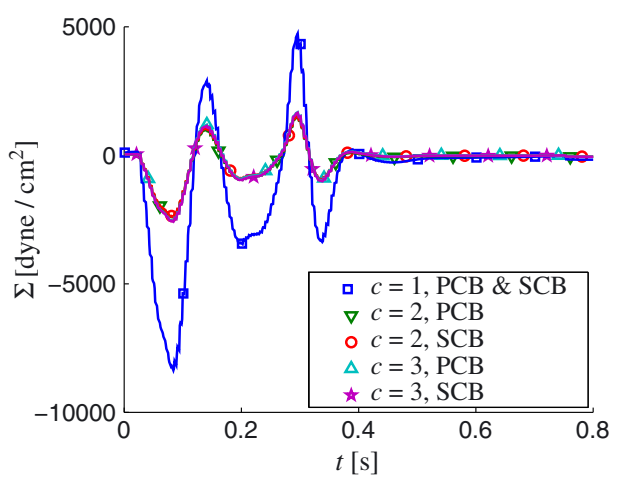

(a)

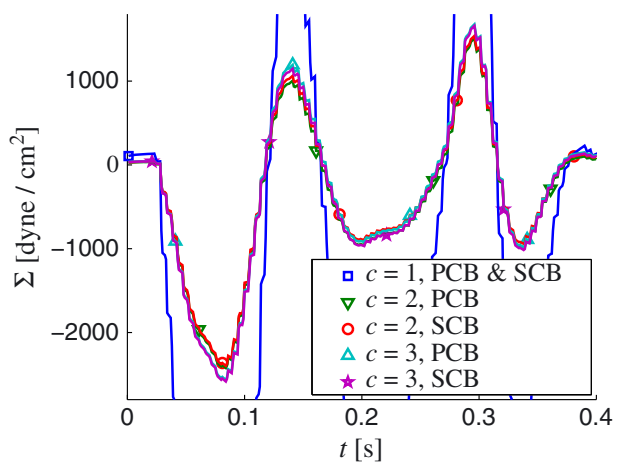

(b)

Figure 11. (a) Coupling stress at different coupling interfaces for the physiological carotid bifurcation (PCB) and the synthetic carotid bifurcation (SCB). (b) Zoomed view of the left graph. 

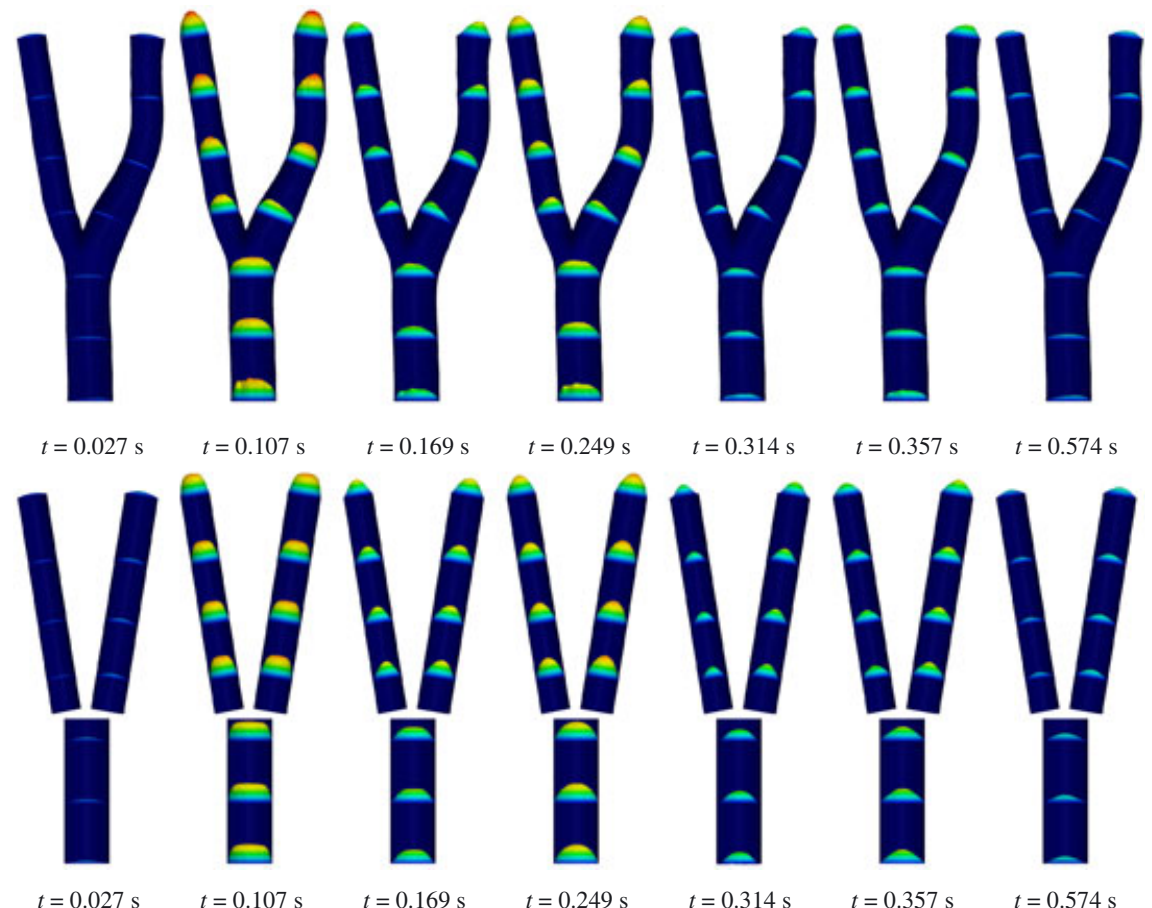

$t=0.169 \mathrm{~s}$

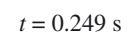

$t=0.314 \mathrm{~s}$

$t=0.357 \mathrm{~s}$

$t=0.574 \mathrm{~s}$

$t=0.027 \mathrm{~s}$
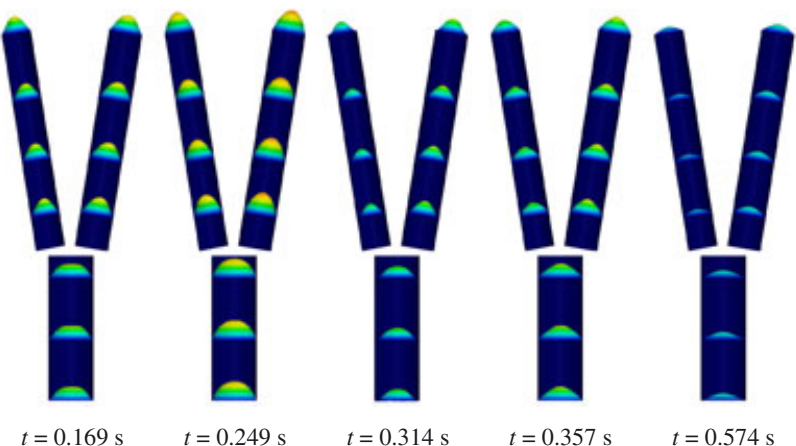

$t=0.314 \mathrm{~s}$

$t=0.357 \mathrm{~s} \quad t=0.574 \mathrm{~s}$

Figure 12. Comparison between the velocity profiles of the PCB (first line), with the ones obtained for the $\mathrm{SCB}$ (second line). Colours: blue $=0.0 \mathrm{~cm} / \mathrm{s}$, red $=79.6 \mathrm{~cm} / \mathrm{s}$.

\subsection{Implementation in a parallel framework}

By the partitioning of the domain discussed in Section 2.1, the original problem is decomposed into $n_{\Omega}$ smaller subproblems defined in $\Omega_{j}$, with $j=1, \ldots, n_{\Omega}$, each of them exchanging information with the others only through the coupling conditions imposed on their artificial boundaries. Unlike the Gauss-Seidel method, which implies a sequential exchange of information at the boundaries, the methods proposed in this paper are perfectly suited for a parallelization of the algorithm at the higher level (i.e. the level of the management of the different subdomains). Let us consider Algorithm 2, which synthetically illustrates the main steps required for the parallel solution of a general problem at each time step.

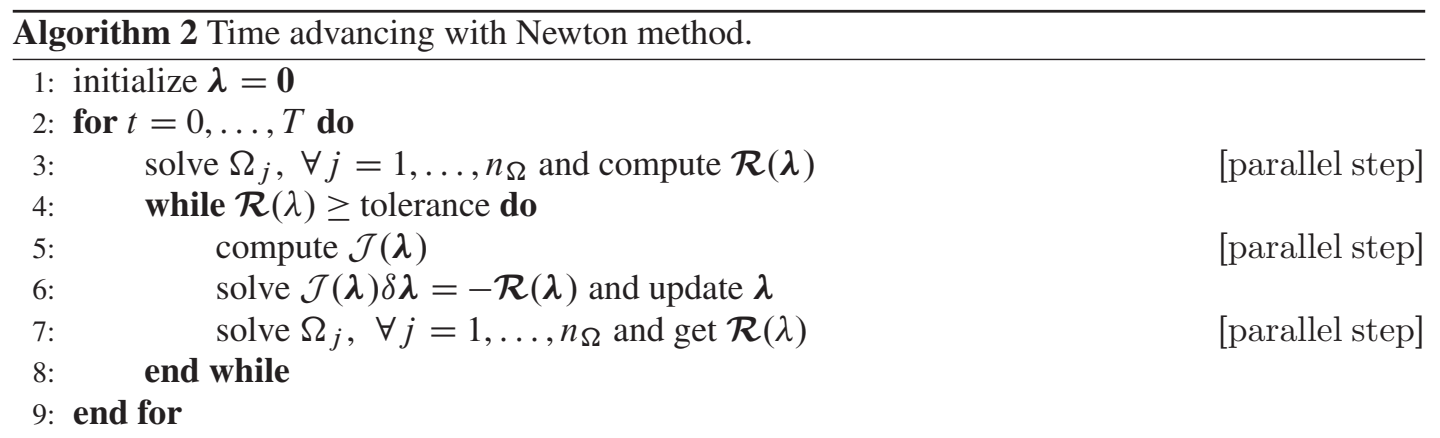

From the computational point of view, the most expensive steps are the solution of the subproblems and the computation of the coefficients of the Jacobian matrix. Nevertheless, all these phases can be easily parallelized. In particular, the solution of the subproblems, performed in steps 3 and 7 , is done for a fixed value of $\lambda$. Hence, each subproblem can be solved on a different machine (where also a lower-level parallelization can be performed), without exchanging any information with the 
others. The same happens for the computation of the coefficients of the Jacobian matrix, performed in step 5. In fact, we can solve each problem, together with the corresponding perturbation on the associated boundary interface, in a completely independent manner. From this analysis, we conclude that the strategies and the algorithms developed in the previous sections can be easily ported to a parallel framework.

\subsection{Geometrical multiscale modelling}

The set of equations in Equation (3) is written in terms of averaged/integrated quantities, and therefore, it can be employed to couple models of different types in a geometrical multiscale framework. Let us consider a problem of wave propagation in a deformable pipe, which can be modelled by the coupling between a 3D fluid-structure interaction (FSI) model (e.g., see [26]) and a non-linear 1D model of fluid flow in compliant vessels (e.g., see [27]), as shown in Figure 13.

In this specific case, strategy $\mathcal{A}$ assumes one of the following forms depending on the assignment of the coupling conditions on the coupling interfaces

$$
\mathcal{A}_{1}:\left\{\begin{array}{l}
Q_{c}=-Q_{c}^{\mathrm{FSI}}\left(\Sigma_{c}\right), \\
\Sigma_{c}=\Sigma_{c}^{1 \mathrm{D}}\left(Q_{c}\right),
\end{array} \quad \mathcal{A}_{2}:\left\{\begin{array}{l}
Q_{c}=-Q_{c}^{1 \mathrm{D}}\left(\Sigma_{c}\right), \\
\Sigma_{c}=\Sigma_{c}^{\mathrm{FSI}}\left(Q_{c}\right),
\end{array}\right.\right.
$$

where $Q_{c}^{\mathrm{FSI}}, Q_{c}^{1 \mathrm{D}}, \Sigma_{c}^{\mathrm{FSI}}$ and $\Sigma_{c}^{1 \mathrm{D}}$ are non-linear operators hiding the complexity of the set of equations of the 1D and FSI models. In particular, strategy $\mathcal{A}_{1}$ corresponds to the imposition of a coupling stress $\Sigma_{c}$ on the FSI model through the operator $Q_{c}^{\mathrm{FSI}}$ and of a flow rate $Q_{c}$ on the 1D model through the operator $\Sigma_{c}^{1 \mathrm{D}}$, whereas strategy $\mathcal{A}_{2}$ is the inverse situation. For strategies $\mathcal{B}$ and $\mathcal{C}$, we have

$$
\mathcal{B}:\left\{\begin{array}{l}
Q_{c}^{\mathrm{FSI}}\left(\Sigma_{c}\right)+Q_{c}=0, \\
Q_{c}^{1 \mathrm{D}}\left(\Sigma_{c}\right)-Q_{c}=0,
\end{array} \quad \mathcal{C}:\left\{\begin{array}{l}
Q_{c, 1}+Q_{c, 2}=0, \\
\Sigma_{c}^{\mathrm{FSI}}\left(Q_{c, 1}\right)-\Sigma_{c}^{1 \mathrm{D}}\left(Q_{c, 2}\right)=0 .
\end{array}\right.\right.
$$

This example allows us to highlight an additional aspect of the coupling strategies described in this paper. From the coupling viewpoint, the models can be seen as black boxes, the coupling boundaries being their unique external interfaces. In addition, from the numerical point of view, all the intricacies related to the nonlinearities of the models are embodied inside these boxes. Naturally, according to the strength of the nonlinearities, we have a different behaviour of the iterative solution method.

\subsection{Preconditioning strategies based on weak coupling}

Suppose that we want to solve a fluid problem in a continuous 3D domain $\Omega$ partitioned into $n_{\Omega}$ smaller subdomains $\Omega_{j}$, with $j=1, \ldots, n_{\Omega}$. Now, using the classical domain decomposition approach described in Section 2.4 (see item 1), we impose the pointwise continuity of the unknowns (velocity and stress) on the coupling interfaces, as shown in Figure 14.

The methodology developed in this work, provides a systematic and simple way to build a preconditioner for this kind of problem. Let us consider a condensation of the coupling quantities through Equation (2) in order to perform a weak coupling on the boundaries, as shown in Figure 15.

This approximation of the original strongly coupled domain decomposition problem (like in Figure 14) is, indeed, the problem, which is addressed throughout this work and that is solved

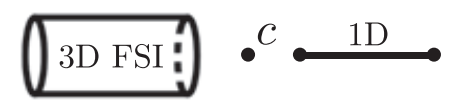

Figure 13. Geometrical multiscale coupling between a fluid-structure interaction (FSI) model and a 1D model. 


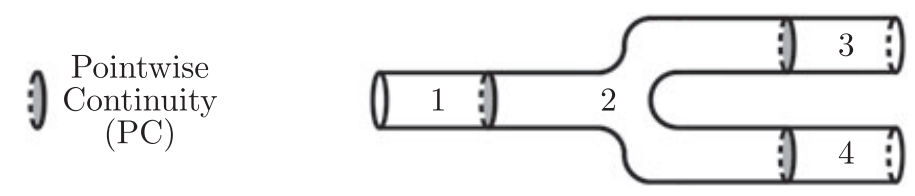

Figure 14. An example of classical domain decomposition, imposing pointwise continuity of the unknowns on the interfaces (in grey). This problem is referred to as $A_{\mathrm{PC}}$.

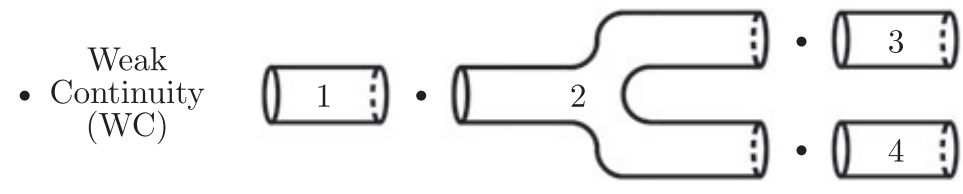

Figure 15. Condensation of the coupling quantities and weak domain decomposition for the problem of Figure 14. This problem is referred to as $A_{\mathrm{WC}}$.

using coupling strategies $\mathcal{A}, \mathcal{B}$ and $\mathcal{C}$, described in Section 2.5. From the mathematical viewpoint, we can define $R$ as the operator that maps the solution computed for the original problem $A_{\mathrm{PC}}$, to the solution for the condensed problem $A_{\mathrm{WC}}$. Hence, we can write

$$
A_{\mathrm{PC}}=R^{T} A_{\mathrm{WC}}
$$

Based on this remark, we can use the solution of problem $A_{\mathrm{WC}}$, which is easier to obtain and whose scalability with respect to the number of subdomains is higher, as a preconditioner for problem $A_{\mathrm{PC}}$. Because all the $n_{\Omega}$ subdomains in Figures 14 and 15 are the same, there exists a trivial map $R$ from the solution of $A_{\mathrm{PC}}$ onto the solution of $A_{\mathrm{WC}}$.

Notice that problem $A_{\mathrm{WC}}$ takes into account the long range interactions between the subdomains through a simpler problem. In other words, $A_{\mathrm{WC}}$ can play the role of a coarse correction for $A_{\mathrm{PC}}$. Moreover, we highlight that the same idea can be employed to introduce a further preconditioner for problem $A_{\mathrm{WC}}$, leading to a two-level preconditioner strategy. This preconditioner may be obtained from a zero-dimensional representation of the problem $($ see $[4,6])$, whose solution gives the approximate values of the coupling quantities on the coupling interfaces.

\section{CONCLUSIONS}

In the present work, the set up of a general decomposition strategy aimed at partitioning dimensionally heterogeneous models into dimensionally homogeneous ones have been investigated within the context of the weak coupling of 3D flow models. In addition, implementation issues related to the application of this methodology have been thoroughly discussed.

Specifically, three strategies for the coupling of multiple 3D flow models have been presented. These strategies are based on a different choice of the boundary conditions to be applied at the interfaces of the subdomains. All of them allow to partition the domain of the 3D Navier-Stokes equations into a network of non-overlapping subdomains, which communicate only through the exchange of averaged/integrated quantities over the interfaces. The coupling equations at the interfaces are based on the conservation of the flow rate and on the continuity of the coupling stress. This choice leads to an approximation of the original problem for the case of 3D Navier-Stokes equations. Moreover, it is perfectly suitable for a geometrical multiscale framework, where most of the couplings involves reduced models, such as OD and 1D models. The numerical schemes employed in this work were the Newton and the Aitken methods. The analysis of the numerical examples conducted here showed that relaxed fixed-point methods behave badly when applied to problems arising in multi-branched systems. In contrast, the Newton method is quite robust for the flow regimes studied here and more generally for closed-loop network configurations. This suggests the use of Newton-like algorithms in order to build convergent schemes. Finally, we also 
showed how the same methodology could be employed to build, in a systematic way, a scalable preconditioner for the original strongly coupled domain decomposition problem.

One of the most appealing aspects of the methodology devised in this paper is the fact that it allows to solve each subdomain separately, leaving all the interactions at the level of the boundary conditions; hence, the implementation in a parallel framework is straightforward and efficient. In addition, no hierarchy has to be established a priori in the setting of the boundary conditions at the branching points. Finally, we also showed how the same methodology could be employed to build, in a systematic way, a scalable preconditioner for the original strongly coupled domain decomposition problem.

\section{ACKNOWLEDGEMENTS}

We acknowledge the European Research Council Advanced Grant 'Mathcard, Mathematical Modelling and Simulation of the Cardiovascular System', Project ERC-2008-AdG 227058. We also acknowledge the support of the Swiss-Brazilian Fund, Project BJRP 011010 (N 590002/2010-4). The second author acknowledges the support of the Brazilian agencies CNPq and FAPERJ. All the numerical results presented in this paper have been computed using the LifeV library (www.lifev.org). The entire LifeV community is kindly acknowledged.

\section{REFERENCES}

1. Blanco PJ, Feijóo RA, Urquiza SA. A unified variational approach for coupling 3D-1D models and its blood flow applications. Comp Meth Appl Mech Engrg 2007; 196(41-44):4391-4410.

2. Blanco PJ, Pivello MR, Urquiza SA, Feijóo RA. On the potentialities of 3D-1D coupled models in hemodynamics simulations. J Biomech 2009; 42(7):919-930.

3. Formaggia L, Gerbeau JF, Nobile F, Quarteroni A. On the coupling of 3D and 1D Navier-Stokes equations for flow problems in compliant vessels. Comp Meth Appl Mech Engrg 2001; 191(6-7):561-582.

4. Formaggia L, Quarteroni A, Veneziani A. Cardiovascular mathematics, Modeling and simulation of the circulatory system, Vol. 1. Springer-Verlag: Milan, 2009.

5. Kim HJ, Vignon-Clementel IE, Figueroa CA, LaDisa JF, Jansen KE, Feinstein JA, Taylor CA. On coupling a lumped parameter heart model and a three-dimensional finite element aorta model. Ann Biomed Eng 2009; 37(11):2153-2169.

6. Migliavacca F, Balossino R, Pennati G, Dubini G, Hsia TY, de Leval MR, Bove EL. Multiscale modelling in biofluidynamics: Application to reconstructive paediatric cardiac surgery. J Biomech 2006; 39(6):1010-1020.

7. Quarteroni A, Veneziani A. Analysis of a geometrical multiscale model based on the coupling of PDE's and ODE's for blood flow simulations. SIAM J Multiscale Model Simul 2003; 1(2):173-195.

8. Urquiza SA, Blanco PJ, Vénere MJ, Feijóo RA. Multidimensional modelling for the carotid artery blood flow. Comp Meth Appl Mech Engrg 2006; 195(33-36):4002-4017.

9. Vignon-Clementel IE, Figueroa CA, Jansen KE, Taylor CA. Outflow boundary conditions for three-dimensional finite element modeling of blood flow and pressure in arteries. Comp Meth Appl Mech Engrg 2006; 195(29-32): 3776-3796.

10. Formaggia L, Gerbeau JF, Nobile F, Quarteroni A. Numerical treatment of defective boundary conditions for the Navier-Stokes equations. SIAM J Numer Anal 2002; 40(1):376-401.

11. Veneziani A, Vergara C. Flow rate defective boundary conditions in haemodynamics simulations. Int J Num Meth Fluids 2005; 47(8-9):803-816.

12. Veneziani A, Vergara C. An approximate method for solving incompressible Navier-Stokes problems with flow rate conditions. Comp Meth Appl Mech Engrg 2007; 196(9-12):1685-1700.

13. Blanco PJ, Feijóo RA, Urquiza SA. A variational approach for coupling kinematically incompatible structural models. Comp Meth Appl Mech Engrg 2008; 197(17-18):1577-1602.

14. Leiva JS, Blanco PJ, Buscaglia GC. Iterative strong coupling of dimensionally-heterogeneous models. Int J Num Meth Engng 2010; 81(12):1558-1580.

15. Blanco PJ, Leiva JS, Feijóo RA, Buscaglia GC. Black-box decomposition approach for computational hemodynamics: one-dimensional models. Comp Meth Appl Mech Engrg 2011; 200(13-16):1389-1405.

16. Ivanova KP, Bournaski EG. Combined distributed and lumped parameters model for transient flow analysis in complex pipe networks. Comp Meth Appl Mech Engrg 1996; 130(1-2):47-56.

17. Kiuchi T. An implicit method for transient gas flows in pipe networks. Int J Heat Fluid Fl 1994; 15(5):378-383.

18. Marin-Artieda CC, Dargush GF. Approximate limit load evaluation of structural frames using linear elastic analysis. Eng Struct 2007; 29(3):296-304.

19. Yang YB, Kuob SR, Wu YS. Incrementally small-deformation theory for nonlinear analysis of structural frames. Eng Struct 2002; 24(6):783-798. 
20. Formaggia L, Lamponi D, Quarteroni A. One-dimensional models for blood flow in arteries. J Eng Math 2003; 47(3-4):251-276.

21. Deparis S. Numerical analysis of axisymmetric flows and methods for fluid-structure interaction arising in blood flow simulation. Ph.D. Thesis, 2004

22. Quarteroni A, Sacco R, Saleri F. Numerical mathematics, Vol. 37. Springer-Verlag: New York, 2000.

23. Sokołowski J, Zolésio J-P. Introduction to shape optimization, Series in computational mathematics, Vol. 16. Springer-Verlag: Berlin and Heidelberg, 1992.

24. Womersley JR. The mathematical analysis of the arterial circulation in a state of oscillatory motion. Technical Report WADC-TR-56-614, Wright Air Dev. Center, 1957.

25. Burman E, Fernández MA, Hansbo P. Continuous interior penalty finite element method for Oseen's equations. SIAM J Numer Anal 2006; 44(3):1248-1274.

26. Deparis S, Discacciati M, Quarteroni A. A domain decomposition framework for fluid-structure interaction problems, Computational Fluid Dynamics. Springer: Berlin, Heidelberg, 2004.

27. Hughes TJR, Lubliner J. On the one-dimensional theory of blood flow in the larger vessels. Math Biosci 1973; 18(1-2):161-170. 